\title{
Phosphine-Mediated Coupling of Gramines with Aldehydes: A Remarkably Simple Synthesis of 3-Vinylindoles
}

\author{
Kam Hung Low and Nabi A. Magomedov \\ Department of Chemistry, University of Rochester, Rochester, NY 14627-0216 \\ magomedov@chem.rochester.edu
}

\section{Supporting Information}

General Information. All reactions were carried out under an atmosphere of nitrogen with magnetic stirring. Solvents were purified prior to use by the Glass Contour solvent purification system. Organic solutions were concentrated in vacuo below $35{ }^{\circ} \mathrm{C}$ at approximately $25 \mathrm{~mm} \mathrm{Hg}$. Purification of reaction products was carried out by flash chromatography using EM Reagent silica gel 60 (230-400 mesh). Analytical thin layer chromatography was performed on EM Reagent $0.25 \mathrm{~mm}$ silica gel $60-\mathrm{F}$ plates. Visualization was accomplished with UV light and potassium permanganate solution or ceric ammonium molybdate solution followed by heating. Melting points were measured with a Bibby Sterilin Stuart SMP10 melting point apparatus and are uncorrected. Infrared spectra were recorded on a Perkin Elmer 1600 series FT-IR spectrometer. ${ }^{1} \mathrm{H}$ NMR spectra were recorded on a Bruker Avance-400 (400 MHz) or Bruker AMX-400 $(400 \mathrm{MHz})$ spectrometer and are reported in ppm using tetramethylsilane as an internal standard $(0.00 \mathrm{ppm})$. Data are reported as $\mathrm{br}=$ broad, $\mathrm{s}=$ singlet, $\mathrm{d}=$ doublet, $\mathrm{t}=$ triplet, $\mathrm{q}$ $=$ quartet, $\mathrm{m}=$ multiplet; coupling constant $(\mathrm{s})$ in $\mathrm{Hz}$; integration. Proton-decoupled ${ }^{13} \mathrm{C}$ NMR spectra were recorded on a Bruker Avance-400 (100 MHz) or Bruker AMX-400 $(100 \mathrm{MHz})$ spectrometer and are reported in ppm using the solvent as the internal standard $\left(\mathrm{CDCl}_{3}\right.$ at $77.23 \mathrm{ppm}, \mathrm{DMSO}-d_{6}$ at $39.52 \mathrm{ppm}, \mathrm{CD}_{3} \mathrm{CN}$ at 1.32 and $\left.118.26 \mathrm{ppm}\right)$. High-resolution mass spectra were obtained on a Thermo Finnigan MAT 95 XL Magnetic Sector spectrometer with electrospray ionization at the State University of New York at Buffalo Mass Spectrometry Facility.

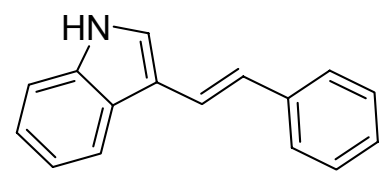

General procedure. To a solution of $174 \mathrm{mg}(1.00 \mathrm{mmol})$ of gramine (1) in $2.0 \mathrm{~mL}$ of dry acetonitrile was added $0.10 \mathrm{~mL}$ of benzaldehyde $(1.00 \mathrm{mmol})$ and $0.37 \mathrm{~mL}$ of $n-\mathrm{Bu}_{3} \mathrm{P}(1.50 \mathrm{mmol})$ at room temperature. The reaction mixture was gently refluxed (oil bath temperature $90{ }^{\circ} \mathrm{C}$ ) for 24 hours after which time TLC (silica gel, 2:1 hexanes/EtOAc) indicated consumption of starting benzaldehyde. The reaction mixture was concentrated in vacuo, taken up in $10 \mathrm{~mL}$ of (3:1 hexanes/EtOAc), filtered through a $2-\mathrm{cm}$ pad of silica gel (the pad washed with $30 \mathrm{~mL}$ of $3: 1$ hexanes/EtOAc), and concentrated in vacuo. The residue was recrystallized $\left(\mathrm{CH}_{2} \mathrm{Cl}_{2} /\right.$ hexane) and the mother liquor was flash chromatographed (hexanes/EtOAc, 4:1) to provide $194 \mathrm{mg}$ (73\% combined yield) of $(E)-3$-(2-phenylethenyl)-1H-indole ${ }^{1}(\mathbf{6})$ as a white crystalline solid: mp 192-193 ${ }^{\circ} \mathrm{C}$ [Lit. ${ }^{l} 193-194{ }^{\circ} \mathrm{C}$ ]; ${ }^{1} \mathrm{H}$ NMR $\left(400 \mathrm{MHz}, \mathrm{CDCl}_{3}\right) \delta 7.14(\mathrm{~d}, J=16.4 \mathrm{~Hz}$, $1 \mathrm{H}), 7.19-7.28(\mathrm{~m}, 4 \mathrm{H}), 7.31-7.41(\mathrm{~m}, 5 \mathrm{H}), 7.52$ (d, $J=7.6 \mathrm{~Hz}, 2 \mathrm{H}), 7.99-8.01$ (d, $J=7.1$ $\mathrm{Hz}, 1 \mathrm{H}), 8.16$ (br s, 1H). 


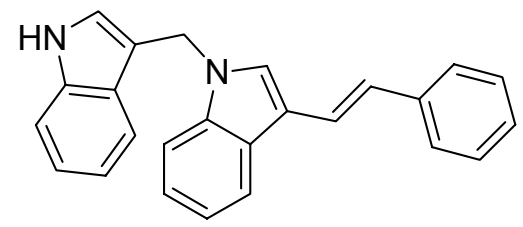

1-((1H-Indol-3-yl)methyl)-3-styryl-1H-indole (7): $\mathrm{mp}$ $159-160{ }^{\circ} \mathrm{C}$; IR $\left(\mathrm{CHCl}_{3}\right) 3409,3051,3032,1632,1597$, $1528,1458,1419,1392,1338,1315,1165,957,741$ $\mathrm{cm}^{-1} ;{ }^{1} \mathrm{H}$ NMR $\left(400 \mathrm{MHz}, \mathrm{CDCl}_{3}\right) \delta 5.41(\mathrm{~s}, 2 \mathrm{H}), 6.97-$ $7.34(\mathrm{~m}, 14 \mathrm{H}), 7.44-7.48(\mathrm{~m}, 4 \mathrm{H}), 7.95$ (br s, 1H), 7.99 $(\mathrm{d}, J=0.7 \mathrm{~Hz}, J=7.3 \mathrm{~Hz}, 1 \mathrm{H}) ;{ }^{13} \mathrm{C}$ NMR $\left(100 \mathrm{MHz}, \mathrm{CDCl}_{3}\right) \delta 42.1,110.2,111.6,111.8$, 114.3, 118.9, 120.4 (2C), 120.6, 122.0, 122.4, 122.8, 123.5, 124.9, 125.8 (2C), 126.6 (2C), 126.7, 127.7, 128.8 (2C), 136.6, 137.5, 138.9; HRMS (DEI): Exact mass calcd for $\mathrm{C}_{25} \mathrm{H}_{20} \mathrm{~N}_{2}\left(\mathrm{M}^{+}\right)$348.1621. Found 348.1610.

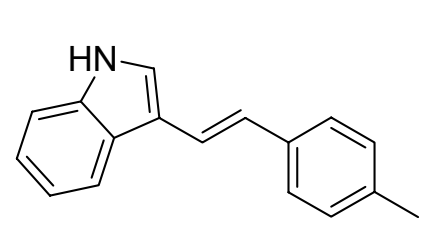

3-(4-Methylstyryl)- $1 \mathrm{H}$-indole $(8){ }^{2}{ }^{2}$ Following General procedure, 8 was obtained in $83 \%$ yield from $174 \mathrm{mg}(1.00$ mmol) of gramine and $0.12 \mathrm{~mL}$ of 4-methylbenzaldehyde (1.00 mmol). Characterization data: colorless solid, $\mathrm{mp} 214-$ $215{ }^{\circ} \mathrm{C}\left(\mathrm{CH}_{2} \mathrm{Cl}_{2} /\right.$ Hexane $)\left[\right.$ Lit. $^{2}$ 215-216 ${ }^{\circ} \mathrm{C}$; ${ }^{1} \mathrm{H}$ NMR $(400$ $\left.\mathrm{MHz}, \mathrm{CDCl}_{3}\right) \delta 2.36(\mathrm{~s}, 3 \mathrm{H}), 7.11(\mathrm{~d}, J=16.5 \mathrm{~Hz}, 1 \mathrm{H}), 7.15-7.30(\mathrm{~m}, 6 \mathrm{H}), 7.34-7.43(\mathrm{~m}$, $4 \mathrm{H}), 7.99(\mathrm{~d}, J=7.4 \mathrm{~Hz}, 1 \mathrm{H}), 8.13(\mathrm{br} \mathrm{s}, 1 \mathrm{H})$.

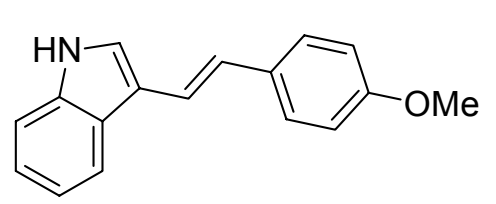

3-(4-Methoxystyryl)-1H-indole (9). ${ }^{1}$ Following General procedure, 9 was obtained in $77 \%$ yield from $174 \mathrm{mg}$ $(1.00 \mathrm{mmol})$ of gramine and $0.13 \mathrm{~mL}$ of $4-$ methoxybenzaldehyde $(1.00 \mathrm{mmol})$. Characterization data: colorless solid, mp 198-199 ${ }^{\circ} \mathrm{C}\left(\mathrm{CH}_{2} \mathrm{Cl}_{2} /\right.$ Hexane $)$ [Lit. ${ }^{1}$ 146-147 $\left.{ }^{\circ} \mathrm{C}\right] ;{ }^{1} \mathrm{H}$ NMR $\left(400 \mathrm{MHz}, \mathrm{CDCl}_{3}\right) \delta 3.84(\mathrm{~s}, 3 \mathrm{H}), 6.91(\mathrm{~d}, J=8.7 \mathrm{~Hz}, 2 \mathrm{H}), 7.09$ (d, $J=16.4 \mathrm{~Hz}, 1 \mathrm{H}), 7.18-7.27(\mathrm{~m}, 4 \mathrm{H}), 7.35(\mathrm{~d}, J=2.5 \mathrm{~Hz}, 1 \mathrm{H}), 7.39(\mathrm{~d}, J=7.1 \mathrm{~Hz}$, $1 \mathrm{H}), 7.46$ (d, $J=8.7 \mathrm{~Hz}, 1 \mathrm{H}), 7.99$ (d, $J=7.2 \mathrm{~Hz}, 1 \mathrm{H}), 8.14$ (br s, $1 \mathrm{H})$.

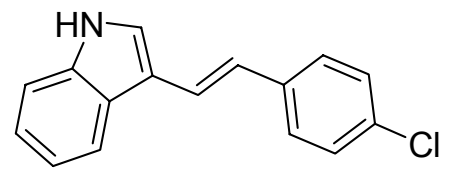

3-(4-Chlorostyryl)-1H-indole (10). ${ }^{2}$ Following General procedure, 10 was obtained in $73 \%$ yield from $174 \mathrm{mg}(1.00$ $\mathrm{mmol}$ ) of gramine and $140 \mathrm{mg}$ of 4-chlorobenzaldehyde $(1.00 \mathrm{mmol})$. Characterization data: white crystalline solid, mp 214-215 ${ }^{\circ} \mathrm{C}\left(\mathrm{CH}_{2} \mathrm{Cl}_{2} / \mathrm{Hexane}\right) ;{ }^{1} \mathrm{H}$ NMR $\left(400 \mathrm{MHz}, \mathrm{CDCl}_{3}\right) \delta 7.08(\mathrm{~d}, J=16.4 \mathrm{~Hz}$, $1 \mathrm{H}), 7.23-7.28(\mathrm{~m}, 4 \mathrm{H}), 7.31(\mathrm{~d}, J=8.2 \mathrm{~Hz}, 2 \mathrm{H}), 7.39(\mathrm{~d}, J=5.4 \mathrm{~Hz}, 1 \mathrm{H}), 7.44(\mathrm{~d}, J=$ $8.4 \mathrm{~Hz}, 2 \mathrm{H}), 7.99$ (d, $J=7.5 \mathrm{~Hz}, 1 \mathrm{H}), 8.20$ (br s, $1 \mathrm{H})$.

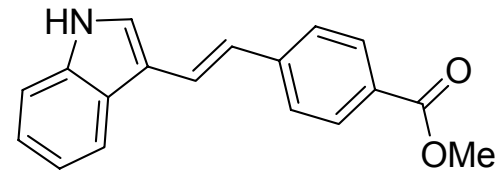

Methyl 4-((E)-2-(1H-indol-3-yl)vinyl)benzoate (11). Following General procedure, 11 was obtained in $80 \%$ yield from $174 \mathrm{mg}(1.00 \mathrm{mmol})$ of gramine and $164 \mathrm{mg}$ of methyl 4-formylbenzoate $(1.00 \mathrm{mmol})$. Characterization data: yellow crystalline solid, $\mathrm{mp} 224-225{ }^{\circ} \mathrm{C}\left(\mathrm{CH}_{2} \mathrm{Cl}_{2} / \mathrm{Hexane}\right)$; IR $\left(\mathrm{CHCl}_{3}\right) 3375,3097$, 3036, 2160, 2029, 1975, 1709, 1632, 1597, 1570, 1528, 1504, 1458, 1427, 1277, 1250, $1177,1107,953,852744 \mathrm{~cm}^{-1} ;{ }^{1} \mathrm{H}$ NMR (400 MHz, DMSO-d $) \delta 3.84(\mathrm{~s}, 3 \mathrm{H}), 7.13-7.18$ $(\mathrm{m}, 3 \mathrm{H}), 7.43(\mathrm{~d}, J=7.8 \mathrm{~Hz}, 1 \mathrm{H}), 7.61(\mathrm{~d}, J=16.5 \mathrm{~Hz}, 1 \mathrm{H}), 7.68-7.72(\mathrm{~m}, 3 \mathrm{H}), 7.91(\mathrm{~d}, J$ $=8.2 \mathrm{~Hz}, 2 \mathrm{H}), 8.04(\mathrm{~d}, J=7.6 \mathrm{~Hz}, 1 \mathrm{H}), 11.46(\mathrm{br} \mathrm{s}, 1 \mathrm{H}) ;{ }^{13} \mathrm{C}$ NMR $(100 \mathrm{MHz}$, DMSO$\left.d_{6}\right) \delta 51.9,112.0,113.5,120.0(2 \mathrm{C}), 121.8,122.0,125.1,125.4(2 \mathrm{C}), 125.7,126.7,127.4$, 
129.6 (2C), 137.1, 143.6, 166.1; HRMS (DEI): Exact mass calcd for $\mathrm{C}_{18} \mathrm{H}_{15} \mathrm{NO}_{2}\left(\mathrm{M}^{+}\right)$ 277.1097. Found: 277.1097.

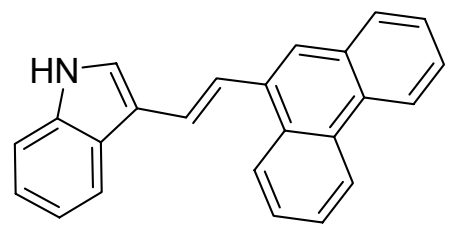

3-((E)-2-(Phenanthren-9-yl)vinyl)-1H-indole

(12). Following General procedure, 12 was obtained in $74 \%$ yield from $174 \mathrm{mg}(1.00 \mathrm{mmol})$ of gramine and $206 \mathrm{mg}$ of 9phenanthrenecarboxyaldehyde $(1.00 \mathrm{mmol})$. Characterization data: yellow crystalline solid, mp 209-211 ${ }^{\circ} \mathrm{C}$ $\left(\mathrm{CH}_{2} \mathrm{Cl}_{2} / \mathrm{Hexane}\right)$; IR $\left(\mathrm{CHCl}_{3}\right) 3414,3055,3036,1632,1528,1454,1419,1385,1338$, 1246, 1099, 953, $741 \mathrm{~cm}^{-1}$; ${ }^{1} \mathrm{H}$ NMR (400 MHz, DMSO- $\left.d_{6}\right) \delta$ 7.20-7.23 (m, 2H), $7.50(\mathrm{~d}$, $J=7.2 \mathrm{~Hz}, 1 \mathrm{H}), 7.60-7.66(\mathrm{~m}, 3 \mathrm{H}), 7.74-7.76(\mathrm{~m}, 2 \mathrm{H}), 7.87-7.91(\mathrm{~m}, 2 \mathrm{H}), 8.04(\mathrm{~d}, J=$ $5.7 \mathrm{~Hz}, 1 \mathrm{H}), 8.13(\mathrm{~d}, J=7.2 \mathrm{~Hz}, 1 \mathrm{H}), 8.18(\mathrm{~s}, 1 \mathrm{H}), 8.79(\mathrm{~d}, J=3.5 \mathrm{~Hz}, 1 \mathrm{H}), 8.89$ (d, $J=$ $5.8 \mathrm{~Hz}, 1 \mathrm{H}), 11.44$ (br s, $1 \mathrm{H}) ;{ }^{13} \mathrm{C}$ NMR (100 MHz, DMSO-d $) \delta 111.9,114.1,119.7$, $119.8,119.8,121.8,122.2,122.7,123.3,124.5,125.3,125.7,126.2,126.4,126.6,126.9$, 126.9, 128.3, 129.1, 130.0, 130.2, 131.7, 134.4, 137.0; HRMS (DEI): Exact mass calcd for $\mathrm{C}_{24} \mathrm{H}_{17} \mathrm{~N}\left(\mathrm{M}^{+}\right)$319.1356. Found 319.1349.

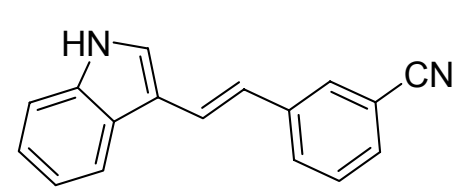

3-((E)-2-(1H-indol-3-yl)vinyl)benzonitrile (13). Following General procedure, 13 was obtained in $71 \%$ yield from 174 $\mathrm{mg}(1.00 \mathrm{mmol})$ of gramine and $131 \mathrm{mg}$ of $3-$ cyanobenzaldehyde $(1.00 \mathrm{mmol})$. Characterization data: yellow crystalline solid, mp $143-144{ }^{\circ} \mathrm{C}\left(\mathrm{CH}_{2} \mathrm{Cl}_{2} / \mathrm{Hexane}\right)$; IR $\left(\mathrm{CHCl}_{3}\right)$ 3410, 3101, 3059, 3036, 2230, 1632, 1589, 1574, 1520, 1477, 1454, 1404, 1323, 1269, 1250, 1223, 1095, 968, 895, 822, 795, $737 \mathrm{~cm}^{-1} ;{ }^{1} \mathrm{H}$ NMR (400 MHz, $\left.\mathrm{CDCl}_{3}\right) \delta 7.08(\mathrm{~d}, J=16.5 \mathrm{~Hz}, 1 \mathrm{H}), 7.19-7.30(\mathrm{~m}, 3 \mathrm{H}), 7.37(\mathrm{~d}, J=16.5 \mathrm{~Hz}, 1 \mathrm{H}), 7.39-$ $7.48(\mathrm{~m}, 4 \mathrm{H}), 7.71(\mathrm{~d}, J=7.3 \mathrm{~Hz}, 1 \mathrm{H}), 7.77(\mathrm{~s}, 1 \mathrm{H}), 7.98$ (d, $J=7.2 \mathrm{~Hz}, 1 \mathrm{H}), 8.28$ (br s, $1 \mathrm{H}) ;{ }^{13} \mathrm{C}$ NMR $\left(100 \mathrm{MHz}, \mathrm{CDCl}_{3}\right) \delta 111.8,113.0,115.2,119.3,120.3,121.1,123.0$, 123.2, 124.7, 125.1, 125.6, 129.3, 129.6, 129.8, 130.0, 137.1, 140.1; HRMS (DEI): Exact mass calcd for $\mathrm{C}_{17} \mathrm{H}_{12} \mathrm{~N}_{2}\left(\mathrm{M}^{+}\right)$244.0995. Found 244.0986.

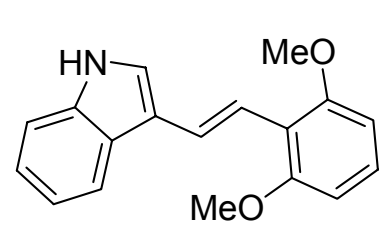

3-(2,6-Dimethoxystyryl)-1H-indole (14). Following General procedure, 14 was obtained in $63 \%$ yield from $174 \mathrm{mg}(1.00$ $\mathrm{mmol}$ ) of gramine and $166 \mathrm{mg}$ of 2,6-dimethoxybenzaldehyde (1.00 mmol). Characterization data: white thick oil; IR $\left(\mathrm{CHCl}_{3}\right)$ 3402, 3051, 3001, 2959, 2939, 2905, 2835, 1701, 1620, 1581, $1454,1431,1338,1250,1107,1034,972,775,741 \mathrm{~cm}^{-1} ;{ }^{1} \mathrm{H} \mathrm{NMR}\left(400 \mathrm{MHz}, \mathrm{CDCl}_{3}\right) \delta$ $3.87(\mathrm{~s}, 6 \mathrm{H}), 6.58(\mathrm{~d}, J=8.0 \mathrm{~Hz}, 2 \mathrm{H}), 7.11(\mathrm{t}, J=8.0 \mathrm{~Hz}, 1 \mathrm{H}), 7.19-7.24(\mathrm{~m}, 4 \mathrm{H}), 7.46$ $(\mathrm{d}, J=16.8 \mathrm{~Hz}, 1 \mathrm{H}), 7.77(\mathrm{~d}, J=16.8 \mathrm{~Hz}, 1 \mathrm{H}), 8.03(\mathrm{br} \mathrm{s}, 2 \mathrm{H}) ;{ }^{13} \mathrm{C}$ NMR $(100 \mathrm{MHz}$, $\left.\mathrm{CDCl}_{3}\right) \delta 56.1,104.4,111.4,116.1,117.2,117.6,120.3,120.6,122.5,123.2,125.7$, 126.1, 127.2, 137.1, 158.5; HRMS (DEI): Exact mass calcd for $\mathrm{C}_{18} \mathrm{H}_{17} \mathrm{NO}_{2}\left(\mathrm{M}^{+}\right)$ 279.1254. Found 279.1251.<smiles>OCc1ccc(/C=C/c2c[nH]c3ccccc23)cc1</smiles>

(4-((E)-2-(1H-indol-3-yl)vinyl)phenyl)methanol (15). Following General procedure, 15 was obtained in $76 \%$ yield from $174 \mathrm{mg}(1.00 \mathrm{mmol})$ of gramine and $136 \mathrm{mg}$ of 4-hydroxymethylbenzaldehyde ${ }^{3} \quad(1.00 \quad \mathrm{mmol})$. 
Characterization data: yellow crystalline solid, mp $212-213{ }^{\circ} \mathrm{C}\left(\mathrm{CH}_{2} \mathrm{Cl}_{2} / \mathrm{Hexane}\right)$; IR $\left(\mathrm{CHCl}_{3}\right)$ 3386, 3283, 3028, 2914, 2871, 1457, 1186, 956, 937, 750, $741 \mathrm{~cm}^{-1} ;{ }^{1} \mathrm{H} \mathrm{NMR}$ $\left(400 \mathrm{MHz}, \mathrm{CD}_{3} \mathrm{CN}\right) \delta 3.14(\mathrm{~s}, 1 \mathrm{H}), 4.57(\mathrm{~s}, 1 \mathrm{H}), 7.12-7.23(\mathrm{~m}, 3 \mathrm{H}), 7.32(\mathrm{~d}, J=8.0 \mathrm{~Hz}$, 2H), $7.40(\mathrm{~d}, J=16.6 \mathrm{~Hz}, 1 \mathrm{H}), 7.46-7.48(\mathrm{~m}, 2 \mathrm{H}), 7.53(\mathrm{~d}, J=8.0 \mathrm{~Hz}, 2 \mathrm{H}), 8.00(\mathrm{~d}, J=$ $7.7 \mathrm{~Hz}, 1 \mathrm{H}), 9.44$ (br s, $1 \mathrm{H}) ;{ }^{13} \mathrm{C} \mathrm{NMR}\left(100 \mathrm{MHz}, \mathrm{CD}_{3} \mathrm{CN}\right) \delta 64.6,112.8,115.5,120.8$, 121.0, 122.9, 123.2, 125.1, 126.2, 126.4, 126.5 (2C), 128.2 (2C), 138.2, 138.7, 141.4; HRMS (DEI): Exact mass calcd for $\mathrm{C}_{17} \mathrm{H}_{15} \mathrm{NO}\left(\mathrm{M}^{+}\right)$249.1148. Found 249.1141.

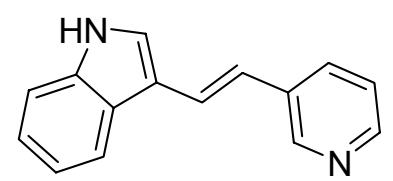

3-((E)-2-(Pyridin-3-yl)vinyl)-1 $\boldsymbol{H}$-indole $\quad$ (16). ${ }^{1} \quad$ Following General procedure, 16 was obtained in $69 \%$ yield from $174 \mathrm{mg}$ (1.00 mmol) of gramine and $0.10 \mathrm{~mL}$ of 3pyridinecarboxaldehyde $(1.00 \mathrm{mmol})$. Characterization data: yellow crystalline solid, mp $194-195{ }^{\circ} \mathrm{C}\left(\mathrm{CH}_{2} \mathrm{Cl}_{2}\right)$ [Lit. ${ }^{1} 195-196{ }^{\circ} \mathrm{C}$ ]; ${ }^{1} \mathrm{H}$ NMR (400 $\left.\mathrm{MHz} \mathrm{CDCl}_{3}\right) \delta 7.09(\mathrm{~d}, J=16.5 \mathrm{~Hz}, 1 \mathrm{H}), 7.25-7.29(\mathrm{~m}, 4 \mathrm{H}), 7.36-7.43(\mathrm{~m}, 3 \mathrm{H}), 7.83(\mathrm{td}$, $J=1.7 \mathrm{~Hz}, J=7.9 \mathrm{~Hz}, 1 \mathrm{H}), 8.00(\mathrm{dd}, J=1.6 \mathrm{~Hz}, J=6.7 \mathrm{~Hz}, 1 \mathrm{H}), 8.38$ (br s, 1H), 8.00 $(\mathrm{dd}, J=1.4 \mathrm{~Hz}, J=4.7 \mathrm{~Hz}, 1 \mathrm{H}), 8.73(\mathrm{~d}, J=1.9 \mathrm{~Hz}, 1 \mathrm{H})$.

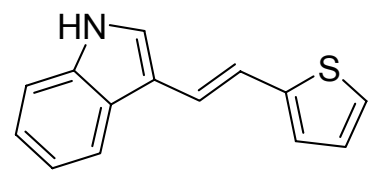

3-((E)-2-(Thiophen-2-yl)vinyl)-1H-indole (17). Following General procedure, 17 was obtained in $87 \%$ yield from $174 \mathrm{mg}$ (1.00 mmol) of gramine and $93 \mu \mathrm{L}$ of 2 -thiophenecarboxaldehyde (1.00 mmol). Characterization data: yellow crystalline solid, $\mathrm{mp}$ 199-200 ${ }^{\circ} \mathrm{C}\left(\mathrm{CH}_{2} \mathrm{Cl}_{2} / \mathrm{Hexane}\right.$, decomp.); IR $\left(\mathrm{CHCl}_{3}\right) 3387,1620,1535,1508,1458,1416$, $1338,1281,1234,1099,949,856,814,748,694 \mathrm{~cm}^{-1} ;{ }^{1} \mathrm{H}$ NMR (400 MHz, DMSO- $\left.d_{6}\right) \delta$ $7.01(\mathrm{~s}, 1 \mathrm{H}), 7.10-7.17(\mathrm{~m}, 4 \mathrm{H}), 7.31-7.33(\mathrm{~m}, 2 \mathrm{H}), 7.41(\mathrm{~d}, J=7.6 \mathrm{~Hz}, 1 \mathrm{H}), 7.64(\mathrm{~s}, 1 \mathrm{H})$, $7.94(\mathrm{~d}, J=7.6 \mathrm{~Hz}, 1 \mathrm{H}), 11.34(\mathrm{~s}, 1 \mathrm{H}) ;{ }^{13} \mathrm{C}$ NMR $\left(100 \mathrm{MHz}, d_{6}\right.$-DMSO) $\delta 111.9,113.1$, 117.1, 119.7, 119.8, 121.8, 122.3, 123.0, 124.3, 124.9, 126.4, 127.8, 137.0, 144.0; HRMS (DEI): Exact mass calcd for $\mathrm{C}_{14} \mathrm{H}_{11} \mathrm{NS}\left(\mathrm{M}^{+}\right)$225.0607. Found 225.0611.

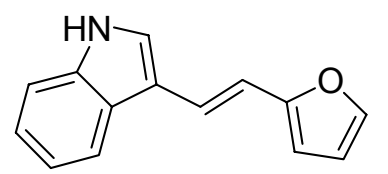

3-((E)-2-(Furan-2-yl)vinyl)-1 $\boldsymbol{H}$-indole $\quad(\mathbf{1 8 )})^{1} \quad$ Following General procedure, 18 was obtained in $76 \%$ yield from $174 \mathrm{mg}$ $(1.00 \mathrm{mmol})$ of gramine and $80 \mu \mathrm{L}$ of 3 -furaldehyde $(1.00 \mathrm{mmol})$. Characterization data: yellow crystalline solid, mp $192-193{ }^{\circ} \mathrm{C}$ $\left(\mathrm{CH}_{2} \mathrm{Cl}_{2} / \mathrm{Hexane}\right) ;{ }^{1} \mathrm{H}$ NMR $\left(400 \mathrm{MHz}, \mathrm{CD}_{3} \mathrm{CN}\right) \delta 6.34(\mathrm{~d}, J=3.1 \mathrm{~Hz}, 1 \mathrm{H}), 6.46(\mathrm{t}, J=$ $1.6 \mathrm{~Hz}, 1 \mathrm{H}), 6.96(\mathrm{~d}, J=16.4 \mathrm{~Hz}, 1 \mathrm{H}), 7.14-7.21(\mathrm{~m}, 2 \mathrm{H}), 7.24(\mathrm{~d}, J=16.4 \mathrm{~Hz}, 1 \mathrm{H})$, 7.45-7.47 (m, 3H), 7.92 (d, $J=7.8 \mathrm{~Hz}, 1 \mathrm{H}), 9.42$ (br s, $1 \mathrm{H})$.

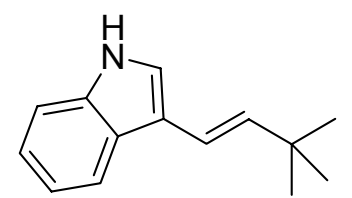

3-((E)-3,3-Dimethylbut-1-enyl)-1H-indole (19). Following General procedure, 19 was obtained in $32 \%$ yield from $174 \mathrm{mg}$ $(1.00 \mathrm{mmol})$ of gramine and $0.57 \mathrm{~mL}$ of pivaldehyde $(5.00 \mathrm{mmol})$. Characterization data: thick colorless oil; IR $\left(\mathrm{CHCl}_{3}\right) 3360,2959$, 2905, 2866, 1705, 1647, 1616, 1531, 1458, 1427, 1362, 1338, 1242, 1103, $744 \mathrm{~cm}^{-1} ;{ }^{1} \mathrm{H}$ NMR (400 MHz, $\left.\mathrm{CDCl}_{3}\right) \delta 1.22(\mathrm{~s}, 9 \mathrm{H}), 6.33(\mathrm{~d}, J=16.3 \mathrm{~Hz}, 1 \mathrm{H})$, $6.53(\mathrm{~d}, J=16.3 \mathrm{~Hz}, 1 \mathrm{H}), 7.17(\mathrm{~d}, J=2.3 \mathrm{~Hz}, 1 \mathrm{H}), 7.24(\mathrm{qd}, J=0.8 \mathrm{~Hz}, J=7.0 \mathrm{~Hz}, 2 \mathrm{H})$, $7.35(\mathrm{~d}, J=7.4 \mathrm{~Hz}, 1 \mathrm{H}), 7.94(\mathrm{~d}, J=7.6 \mathrm{~Hz}, 1 \mathrm{H}), 7.95($ br s, $1 \mathrm{H}) ;{ }^{13} \mathrm{C}$ NMR $(100 \mathrm{MHz}$, $\left.\mathrm{CDCl}_{3}\right) \delta 30.1,33.7,111.5,115.7,117.0,120.1,120.2,122.3,122.5,125.9,136.9,139.5$ HRMS (DEI): Exact mass calcd for $\mathrm{C}_{14} \mathrm{H}_{17} \mathrm{~N}\left(\mathrm{M}^{+}\right)$199.1356. Found 199.1353. 


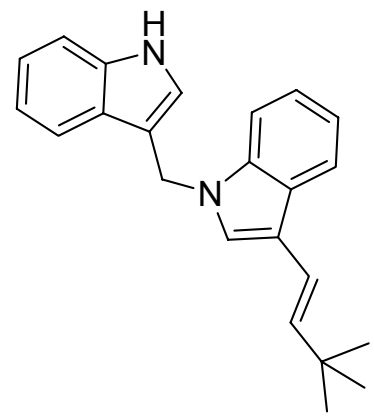

1-((1H-Indol-3-yl)methyl)-3-((E)-3,3-dimethylbut-1-enyl)-1Hindole was isolated in $15 \%$ yield. Characterization data: white solid, mp $75-76{ }^{\circ} \mathrm{C}$ (Hexane/Ethyl acetate); IR $\left(\mathrm{CHCl}_{3}\right) 3348$, 2956, 2916, 2850, 1701, 1612, 1462, 1354, 1246, 1173, 1099, $744 \mathrm{~cm}^{-1} ;{ }^{1} \mathrm{H}$ NMR $\left(400 \mathrm{MHz}, \mathrm{CDCl}_{3}\right) \delta 1.11(\mathrm{~s}, 9 \mathrm{H}), 5.33$, (s, $2 \mathrm{H}), 6.20(\mathrm{~d}, J=16.4 \mathrm{~Hz}, 1 \mathrm{H}), 6.43(\mathrm{~d}, J=16.4 \mathrm{~Hz}, 1 \mathrm{H}), 6.82(\mathrm{~d}$, $J=1.8 \mathrm{~Hz}, 1 \mathrm{H}), 7.04-7.08(\mathrm{~m}, 2 \mathrm{H}), 7.14-7.26(\mathrm{~m}, 4 \mathrm{H}), 7.39$ (d, $J$ $=7.9 \mathrm{~Hz}, 1 \mathrm{H}), 7.45(\mathrm{~d}, J=7.9 \mathrm{~Hz}, 1 \mathrm{H}), 7.81(\mathrm{br} \mathrm{s}, 1 \mathrm{H}), 7.88(\mathrm{~d}, J$ $=7.8 \mathrm{~Hz}, 1 \mathrm{H}) ;{ }^{13} \mathrm{C}$ NMR $\left(100 \mathrm{MHz}, \mathrm{CDCl}_{3}\right) \delta 30.1,33.6,41.9$, $110.0,111.5,112.1,114.2,117.1,118.9,119.8,120.2,120.4,122.0,122.7,123.4,126.1$, 126.6, 126.7, 136.5, 137.3, 138.8; HRMS (DEI): Exact mass calcd for $\mathrm{C}_{23} \mathrm{H}_{24} \mathrm{~N}_{2}\left(\mathrm{M}^{+}\right)$ 328.1934. Found 328.1928.

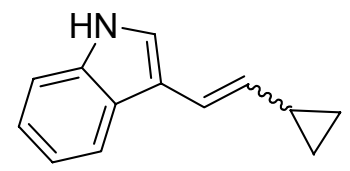

3-(2-Cyclopropylvinyl)-1H-indole (20). Following General procedure, 20 was obtained in 55\% yield $(E: Z=71: 29)$ from 174 $\mathrm{mg} \quad(1.00 \mathrm{mmol})$ of gramine and $0.11 \mathrm{~mL}$ of cyclopropanecarbaldehyde $(1.50 \mathrm{mmol})$. Characterization data: yellow amorphous solid; IR $\left(\mathrm{CHCl}_{3}\right)$ 3392, 3074, 3051, 2999, 1531, 1458, 1423, 1230, $1095,949,798,741 \mathrm{~cm}^{-1} . \boldsymbol{E - 2 0}:{ }^{1} \mathrm{H}$ NMR $\left(400 \mathrm{MHz}, \mathrm{CD}_{3} \mathrm{CN}\right) \delta 0.49-0.52(\mathrm{~m}, 2 \mathrm{H}), 0.78-$ $0.81(\mathrm{~m}, 2 \mathrm{H}), 1.56-1.58(\mathrm{~m}, 1 \mathrm{H}), 5.76(\mathrm{dd}, J=8.7 \mathrm{~Hz}, J=16.0 \mathrm{~Hz}, 1 \mathrm{H}), 6.67(\mathrm{~d}, J=16.0$ $\mathrm{Hz}, 1 \mathrm{H}), 7.08-7.25(\mathrm{~m}, 2 \mathrm{H}), 7.42-7.46(\mathrm{~m}, 2 \mathrm{H}), 7.79$ (d, J=7.9 Hz, 1H), 9.08 (br s, 1H); ${ }^{13} \mathrm{C}$ NMR $\left(100 \mathrm{MHz}, \mathrm{CD}_{3} \mathrm{CN}\right) \delta 7.2,15.4,112.4,115.3,120.3,120.4,121.0,122.7$, 123.7, 126.2, 131.5, 137.8; HRMS (DEI): Exact mass calcd for $\mathrm{C}_{13} \mathrm{H}_{13} \mathrm{~N}\left(\mathrm{M}^{+}\right)$183.1043. Found 183.1030.

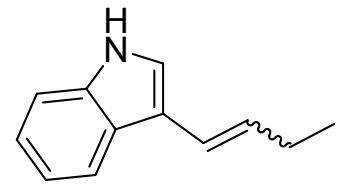

3-(But-1-enyl)-1H-indole (21). Following General procedure, 21 was obtained in $11 \%$ yield $(E: Z=86: 14)$ from $174 \mathrm{mg}(1.00 \mathrm{mmol})$ of gramine and $0.40 \mathrm{~mL}$ of propanal $(5.00 \mathrm{mmol})$ as an amorphous solid: IR $\left(\mathrm{CHCl}_{3}\right)$ 3389, 2960, 2930, 2871, 1456, 1249, 1099, 957, 750, $741 \mathrm{~cm}^{-1} . \boldsymbol{E}-\mathbf{2 1}:{ }^{1} \mathrm{H}$ NMR $\left(400 \mathrm{MHz}, \mathrm{DMSO}-d_{6}\right) \delta 1.08(\mathrm{t}, J=7.4 \mathrm{~Hz}, 3 \mathrm{H}), 2.21$ (q, $J=7.2 \mathrm{~Hz}, 2 \mathrm{H}), 6.16(\mathrm{td}, J=6.5 \mathrm{~Hz}, J=16.0 \mathrm{~Hz}, 1 \mathrm{H}), 6.53(\mathrm{~d}, J=16.0 \mathrm{~Hz}, 1 \mathrm{H}), 7.03(\mathrm{t}$, $J=7.2 \mathrm{~Hz}, 1 \mathrm{H}), 7.10(\mathrm{t}, J=7.7 \mathrm{~Hz}, 1 \mathrm{H}), 7.36-7.40(\mathrm{~m}, 2 \mathrm{H}), 7.76(\mathrm{~d}, J=7.9 \mathrm{~Hz}, 1 \mathrm{H})$, 11.06 (br s, $1 \mathrm{H}) ;{ }^{13} \mathrm{C}$ NMR $\left(100 \mathrm{MHz}, \mathrm{DMSO}-d_{6}\right) \delta 14.6,26.5,112.0,113.9,119.5$, 119.8, 121.7, 122.5, 124.3, 125.6, 127.7, 137.2; HRMS (DEI): Exact mass calcd for $\mathrm{C}_{12} \mathrm{H}_{13} \mathrm{~N}\left(\mathrm{M}^{+}\right)$171.1043. Found 171.1042.

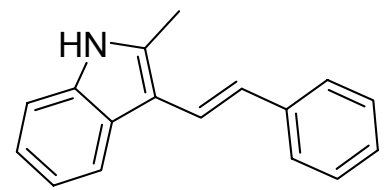

2-Methyl-3-styryl-1H-indole (23). Following General procedure, 23 was obtained in 55\% yield from $140 \mathrm{mg}(0.74$ mmol) of 2-methylgramine (22) ${ }^{4}$ and $74 \mu \mathrm{L}$ of benzaldehyde (0.74 mmol). Characterization data: yellow crystalline solid, mp $190-191{ }^{\circ} \mathrm{C}\left(\mathrm{CH}_{2} \mathrm{Cl}_{2} / \mathrm{Hexane}\right)$; IR $\left(\mathrm{CHCl}_{3}\right) 3402,3032,2912,1632,1458,1427,1308$, 1246, 953, $741 \mathrm{~cm}^{-1} ;{ }^{1} \mathrm{H} \mathrm{NMR}\left(400 \mathrm{MHz}, \mathrm{CDCl}_{3}\right) \delta 2.36(\mathrm{~s}, 3 \mathrm{H}), 7.07$ (d, $J=16.4 \mathrm{~Hz}$, 1H), 7.15-7.21 (m, 4H), 7.25 (d, $J=16.4 \mathrm{~Hz}, 1 \mathrm{H}), 7.33$ (t, $J=7.6 \mathrm{~Hz}, 2 \mathrm{H}), 7.50(\mathrm{~d}, J=$ $7.6 \mathrm{~Hz}, 2 \mathrm{H}), 7.61$ (br s, $1 \mathrm{H}), 7.94(\mathrm{~d}, J=7.1 \mathrm{~Hz}, 1 \mathrm{H}) ;{ }^{13} \mathrm{C}$ NMR $\left(100 \mathrm{MHz}, \mathrm{CDCl}_{3}\right) \delta$ $12.4,110.8,111.1,119.8,120.6,121.8,121.9,125.4,125.8,126.6,126.7,128.8,134.8$, 
135.7, 139.2; HRMS (DEI): Exact mass calcd for $\mathrm{C}_{17} \mathrm{H}_{15} \mathrm{~N}\left(\mathrm{M}^{+}\right)$233.1199. Found 233.1199 .

${ }^{1}$ De Silva, S. O.; Snieckus, V. Can. J. Chem. 1974, 52, 1294-1306

2 Tewari, R. S.; Gupta, K. C. Indian J. Chem., Sect B 1976, 14B, 419-421.

${ }^{3}$ Mak, C. C.; Bampos, N.; Darling, S. L.; Montalti, M.; Prodi, L.; Sanders, J. K. M. J. Org. Chem. 2001, 66, 4476-4486.

${ }^{4}$ Bennett, R.; Maggiolo, A.; Shah, T. J. Heterocycl. Chem. 1981, 18, 391-394. 


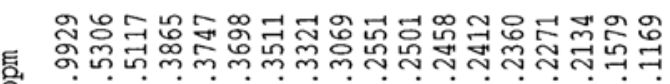

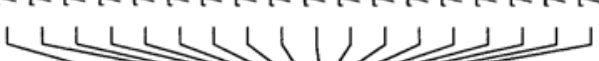

게 $\rightarrow$ th

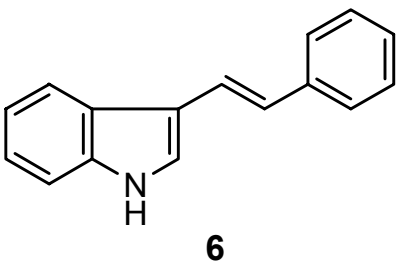

$\mathrm{CDCl}_{3}, 400 \mathrm{MHz}$

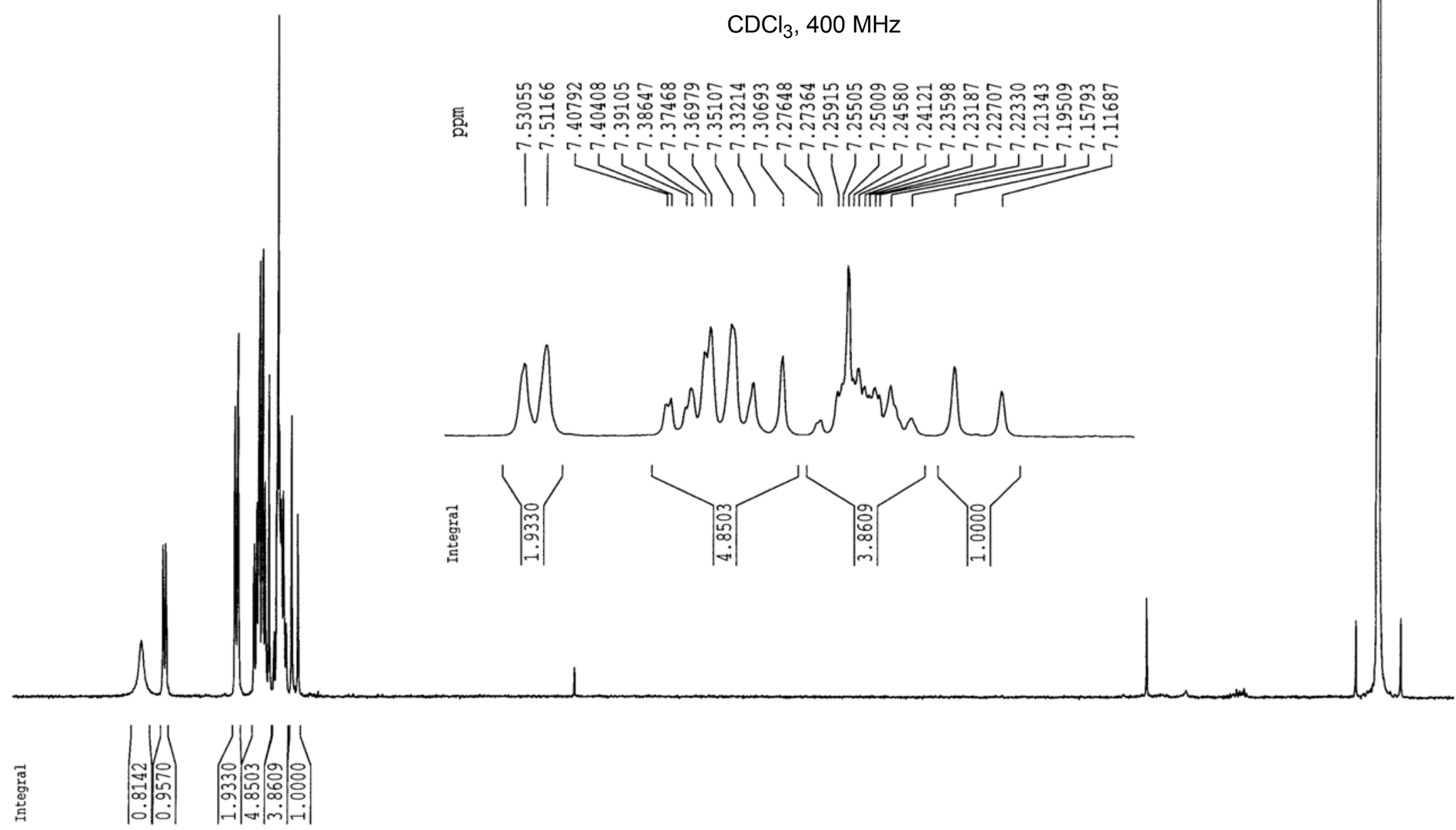

$\begin{array}{llllllllll}\mathrm{ppm} & 8 & 7 & 6 & 5 & 4 & 3 & 2 & 1 & 0\end{array}$


镸

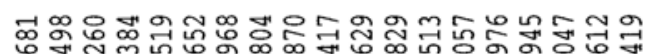

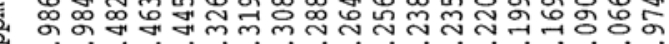

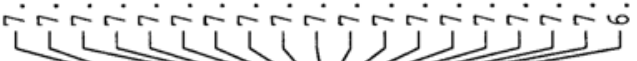

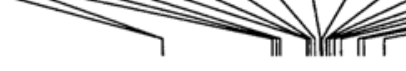

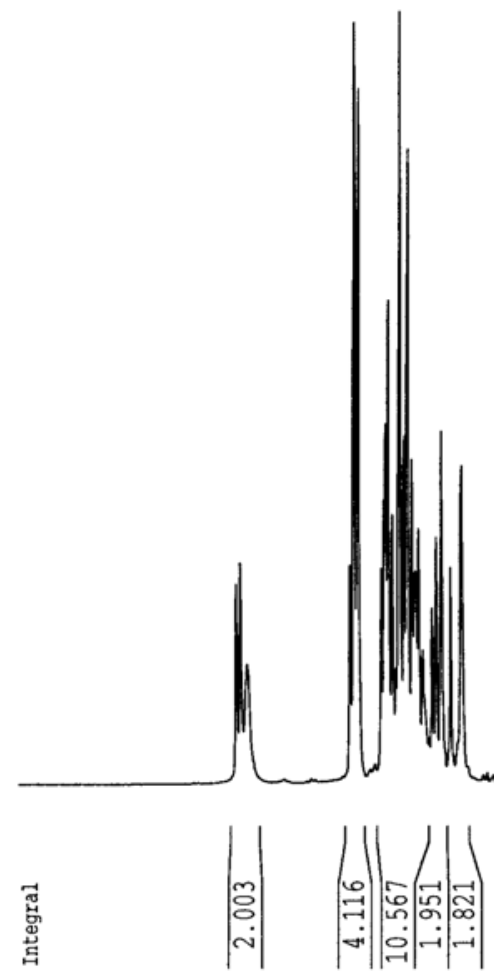

ppm 8

8

7

6

5

5

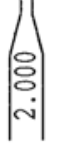

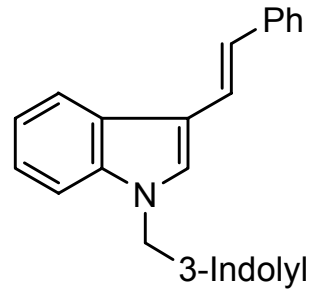

$\mathrm{CDCl}_{3}, 400 \mathrm{MHz}$

⿷

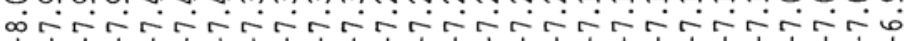

(I) $n$ mो
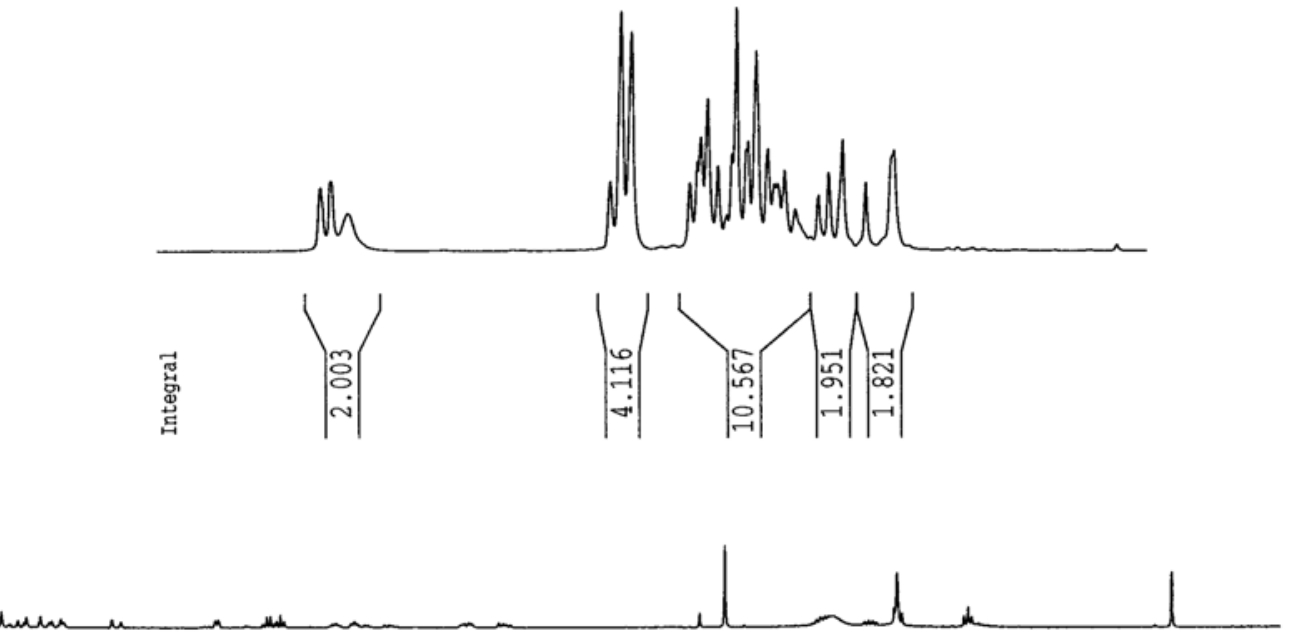


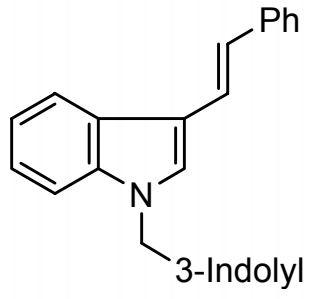

7

$\mathrm{CDCl}_{3}, 100 \mathrm{MHz}$

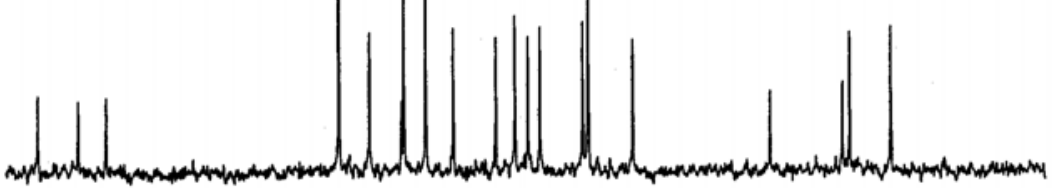

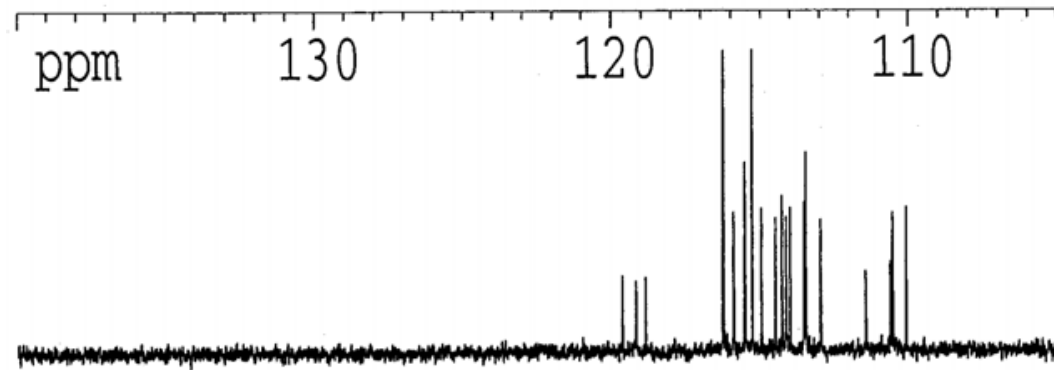

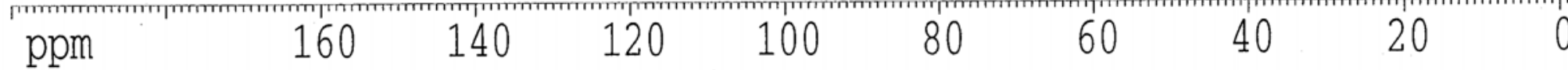




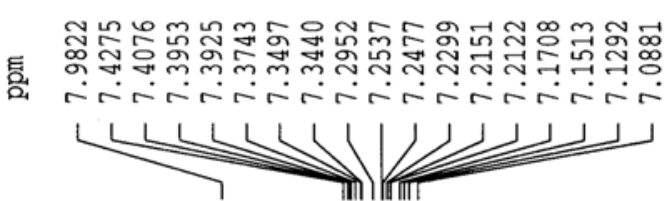

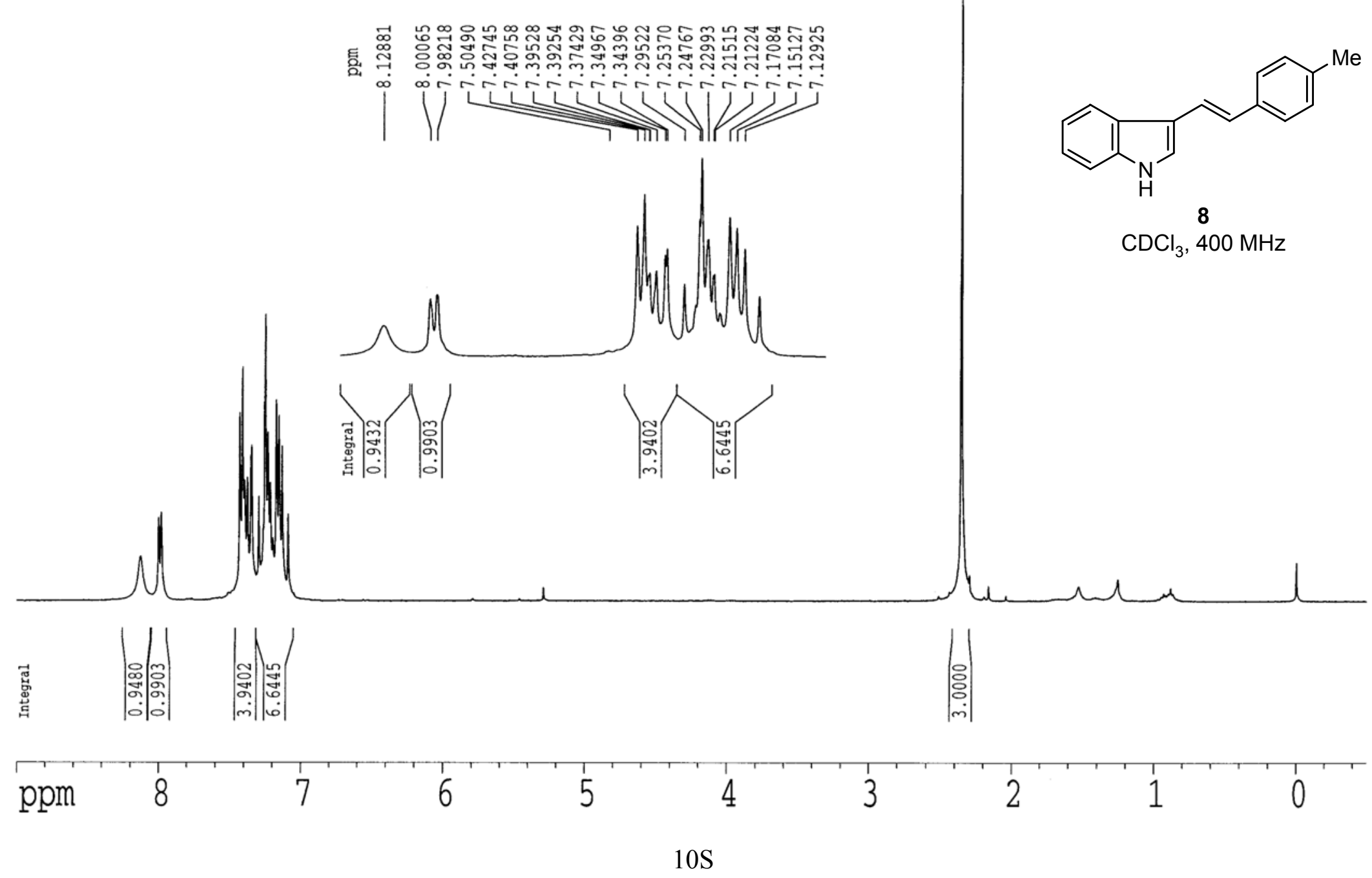




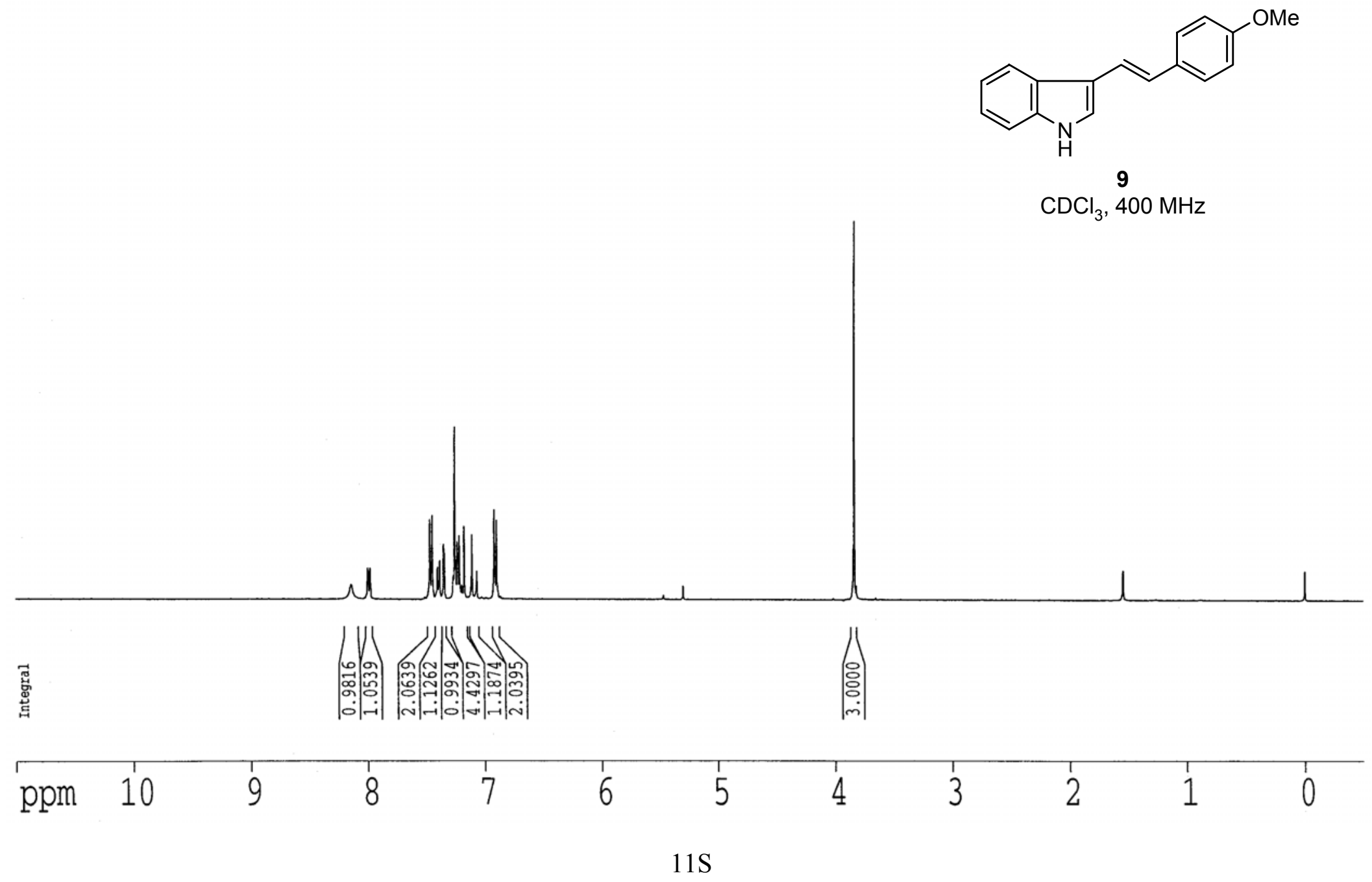




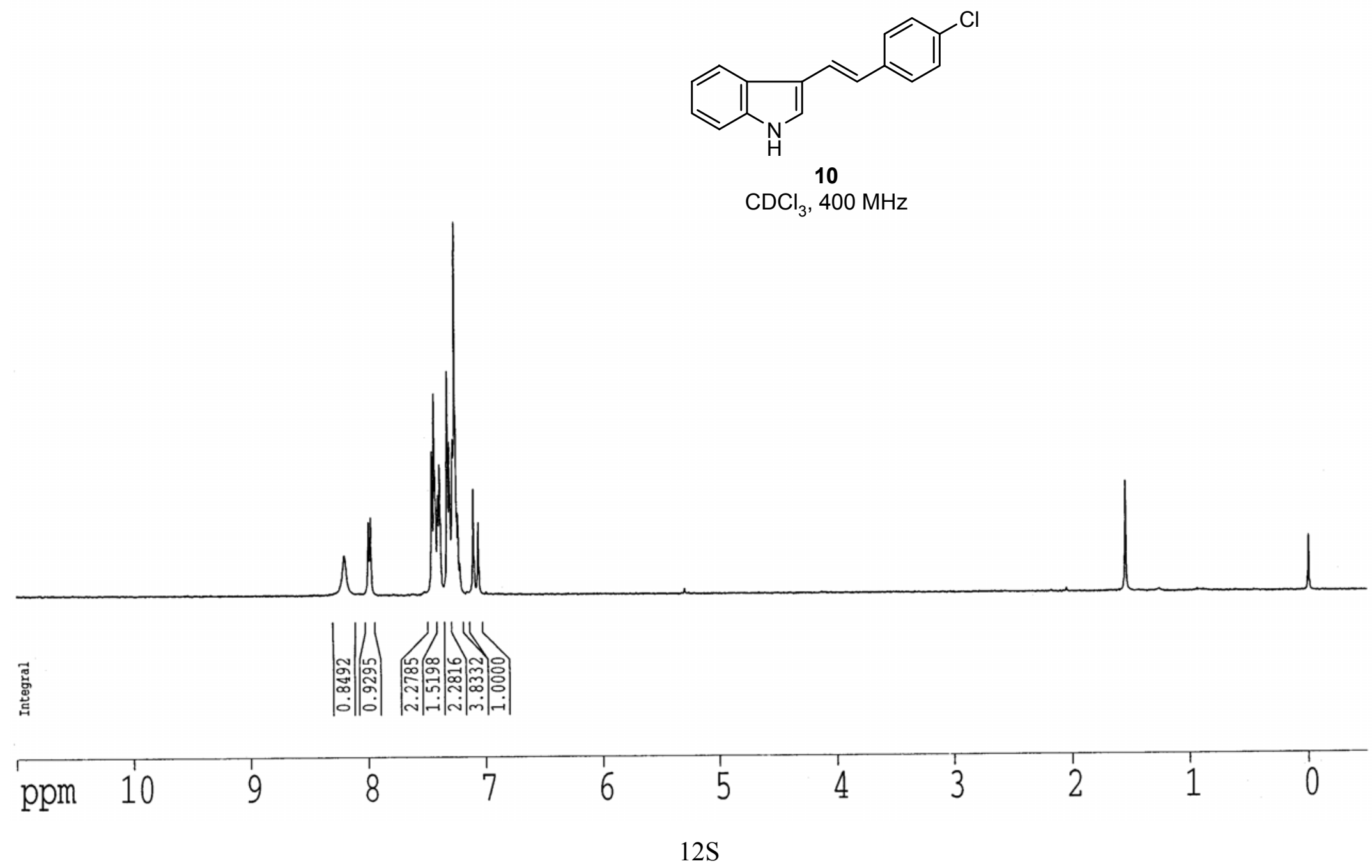


$\stackrel{2}{1 !}$

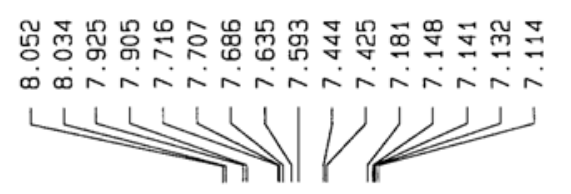

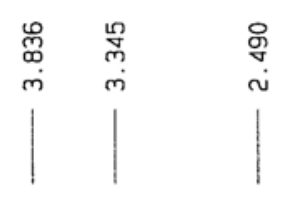

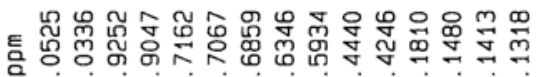
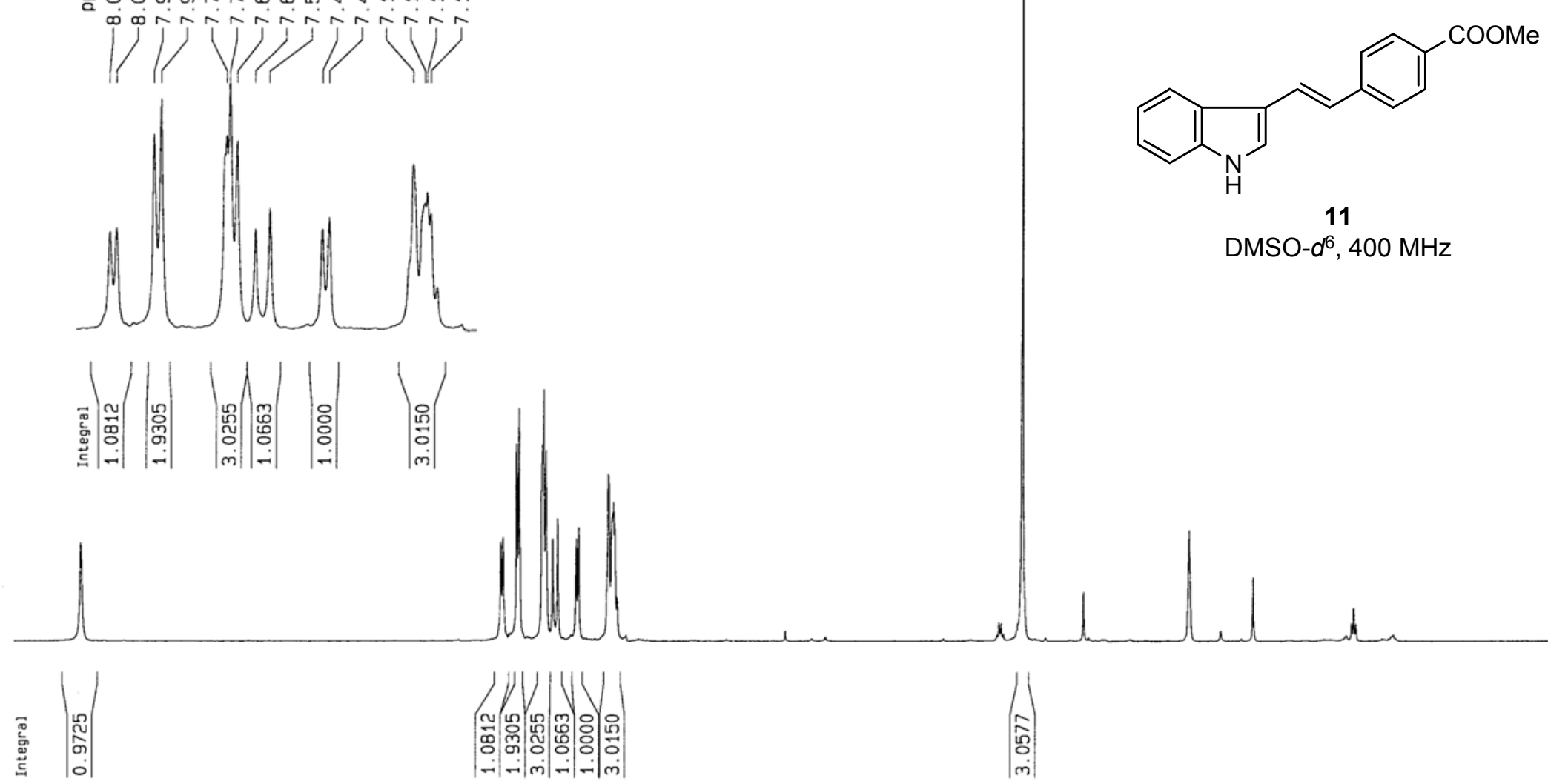

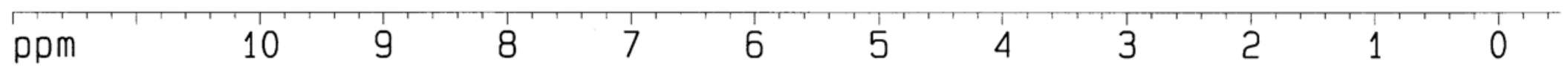




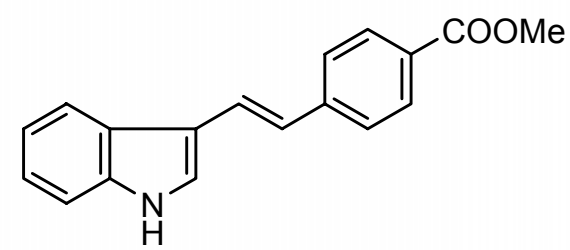

$$
\text { DMSO- } d^{6}, 100 \mathrm{MHz}
$$

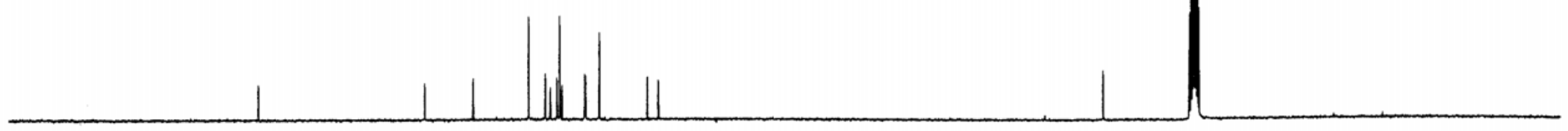

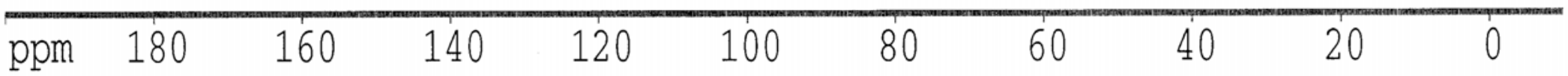




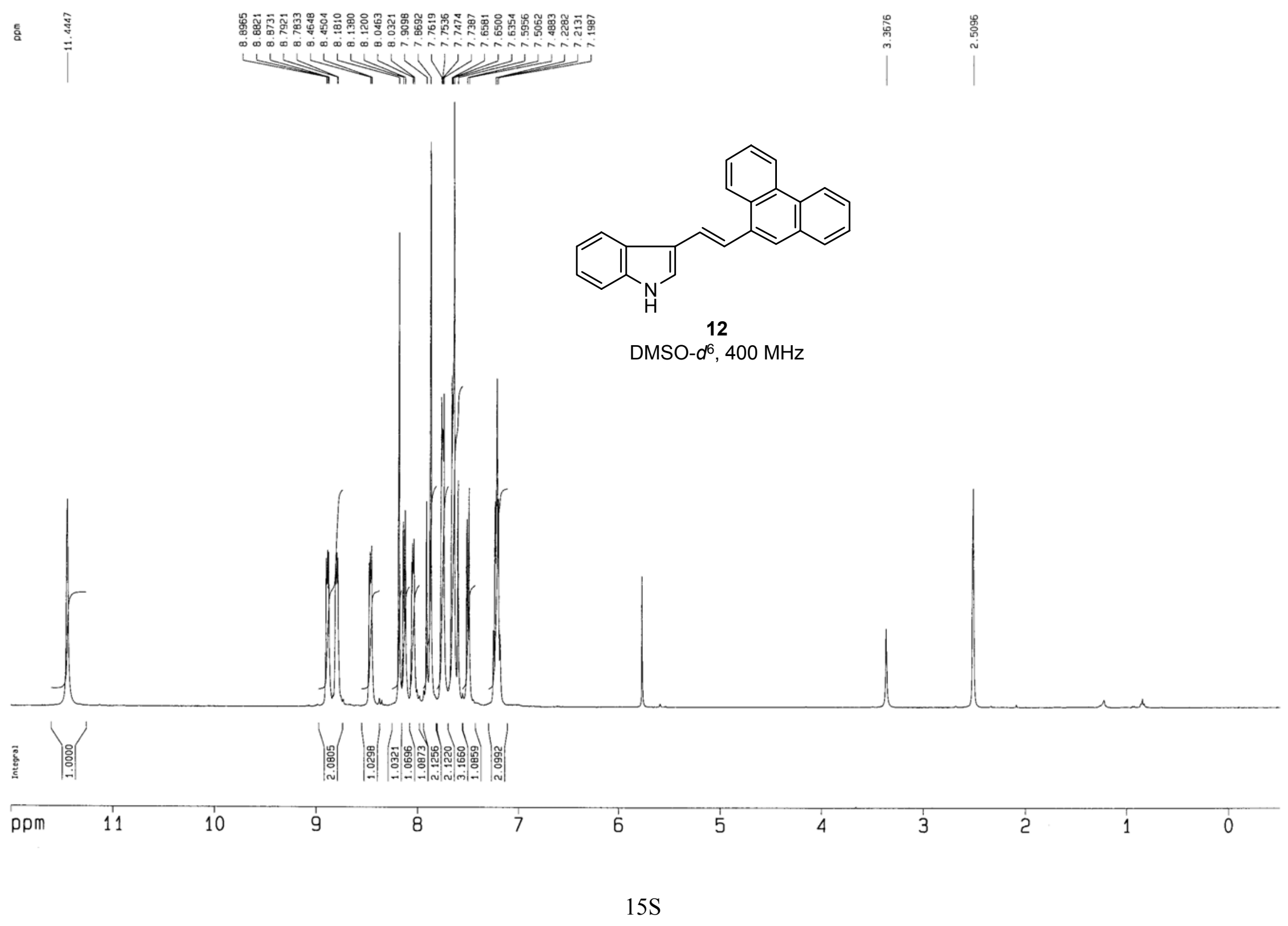




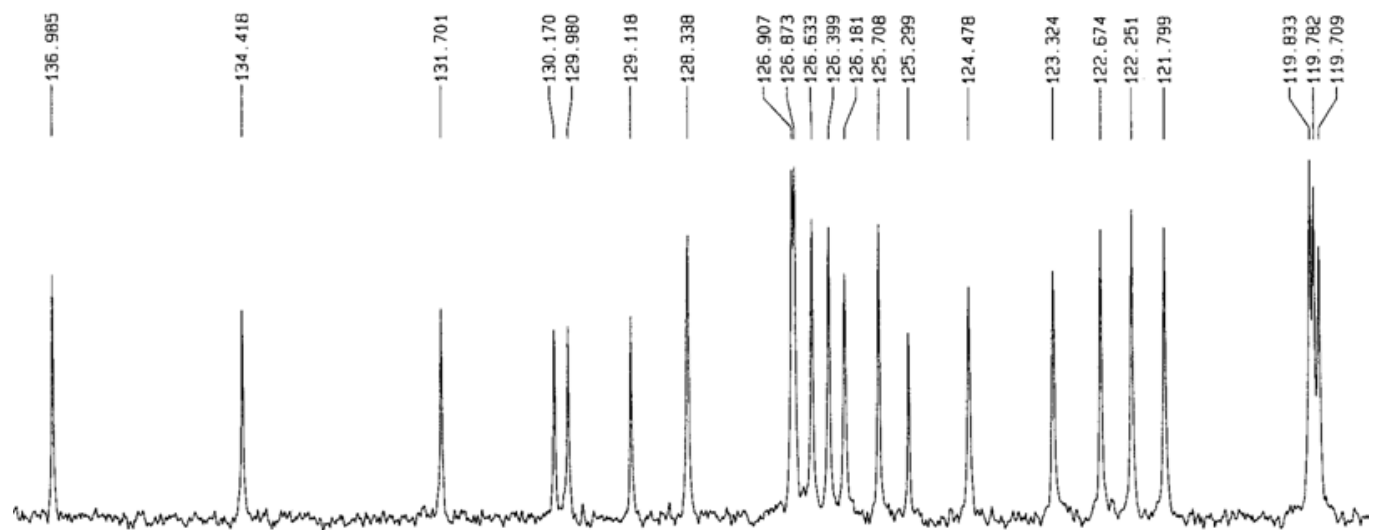

DMSO- $d^{6}, 100 \mathrm{MHz}$

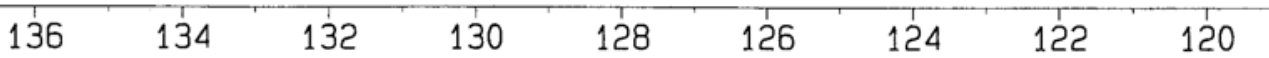



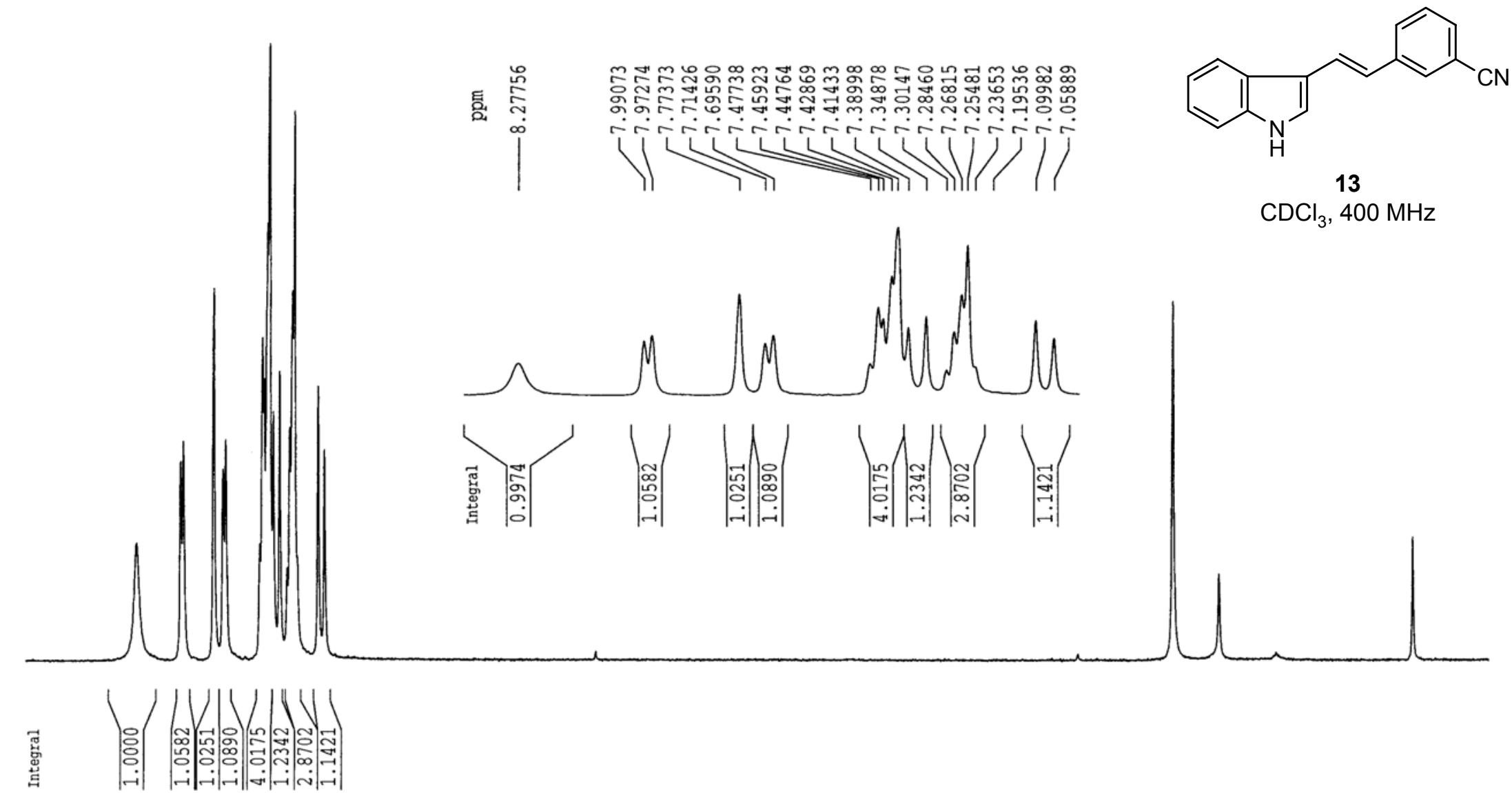

\begin{tabular}{llllllllll}
\hline ppm & 8 & 7 & 6 & 5 & 4 & 3 & 2 & 1 & 0 \\
& & & & & & & & &
\end{tabular}




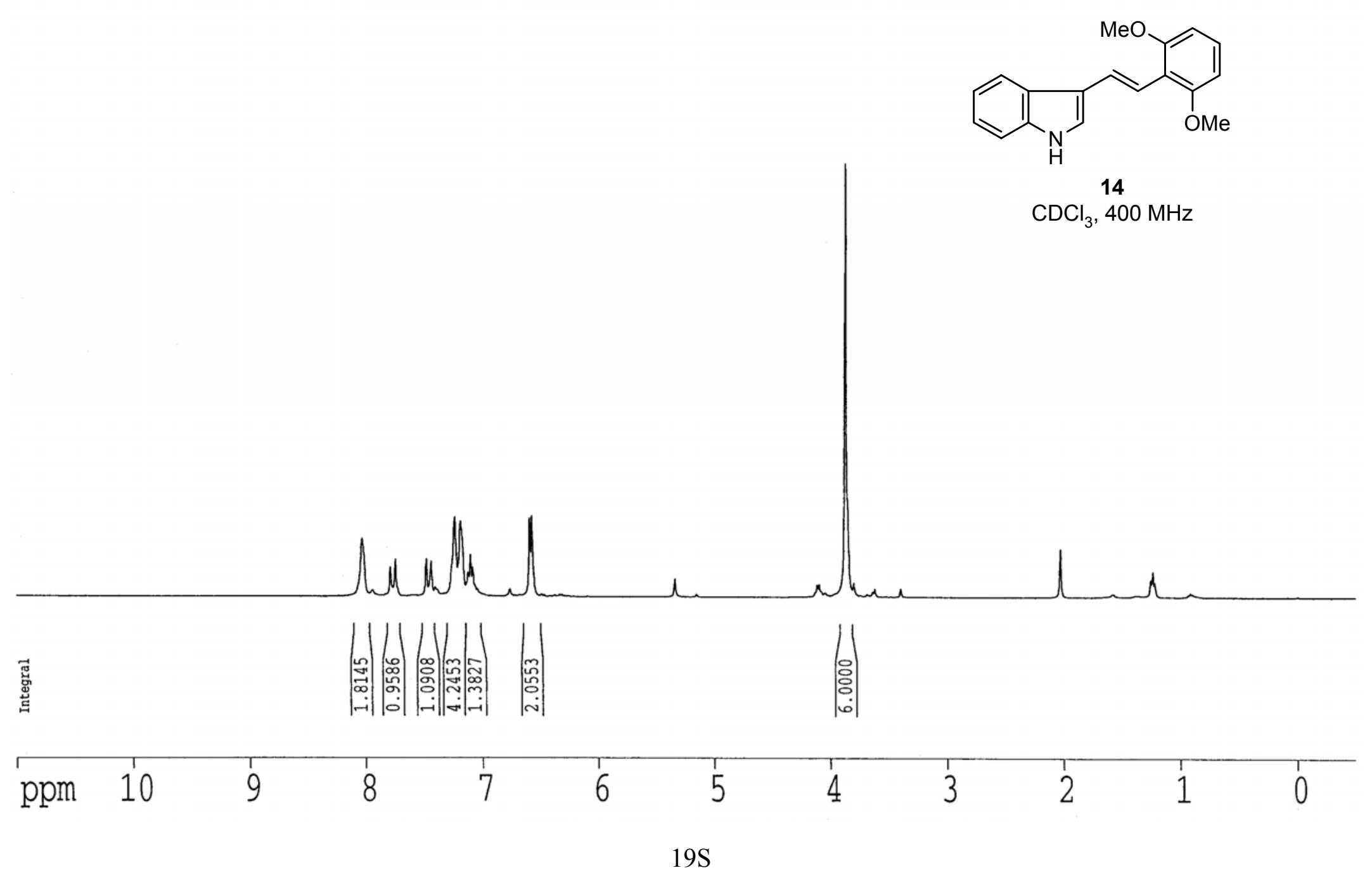




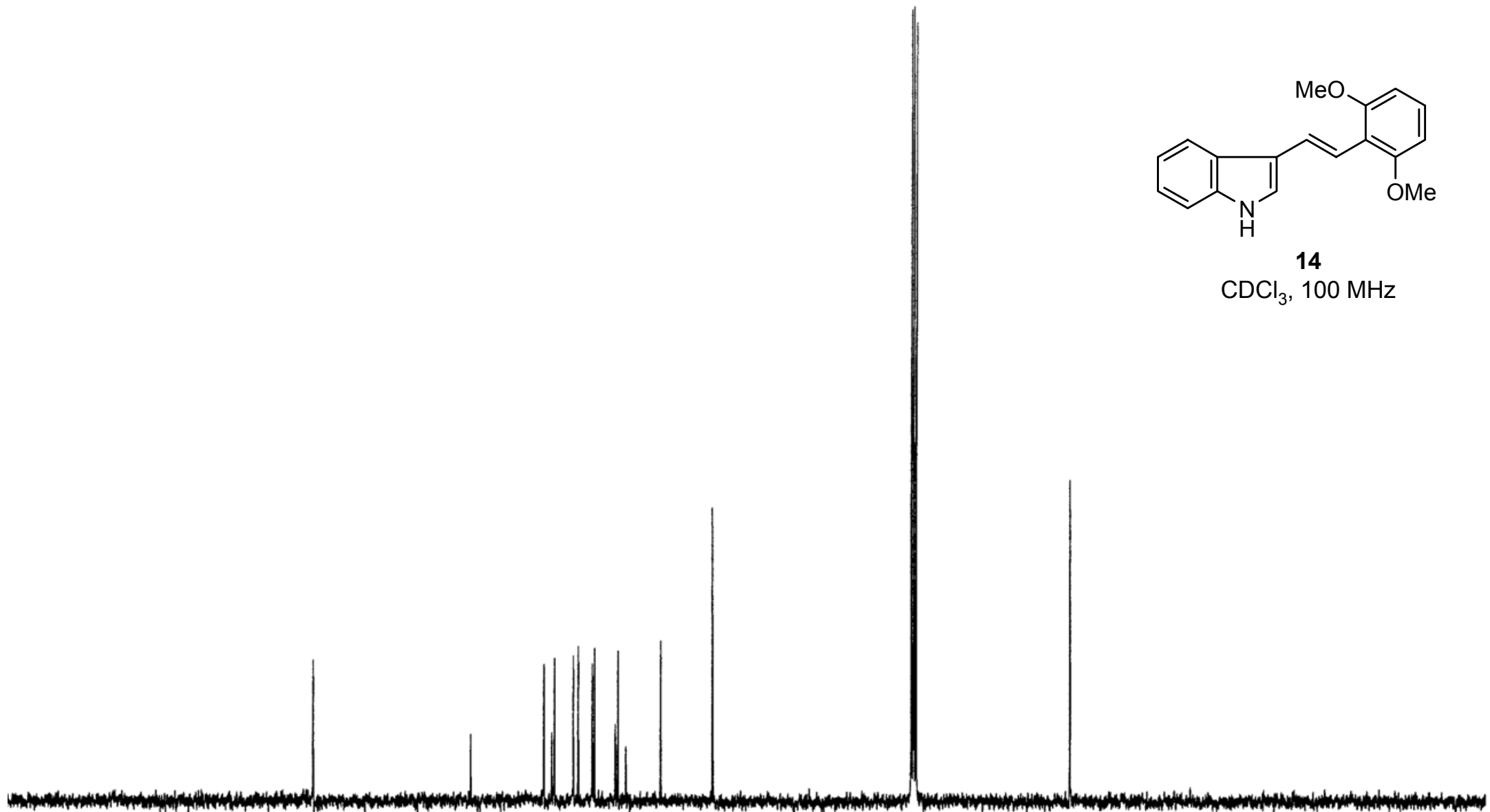



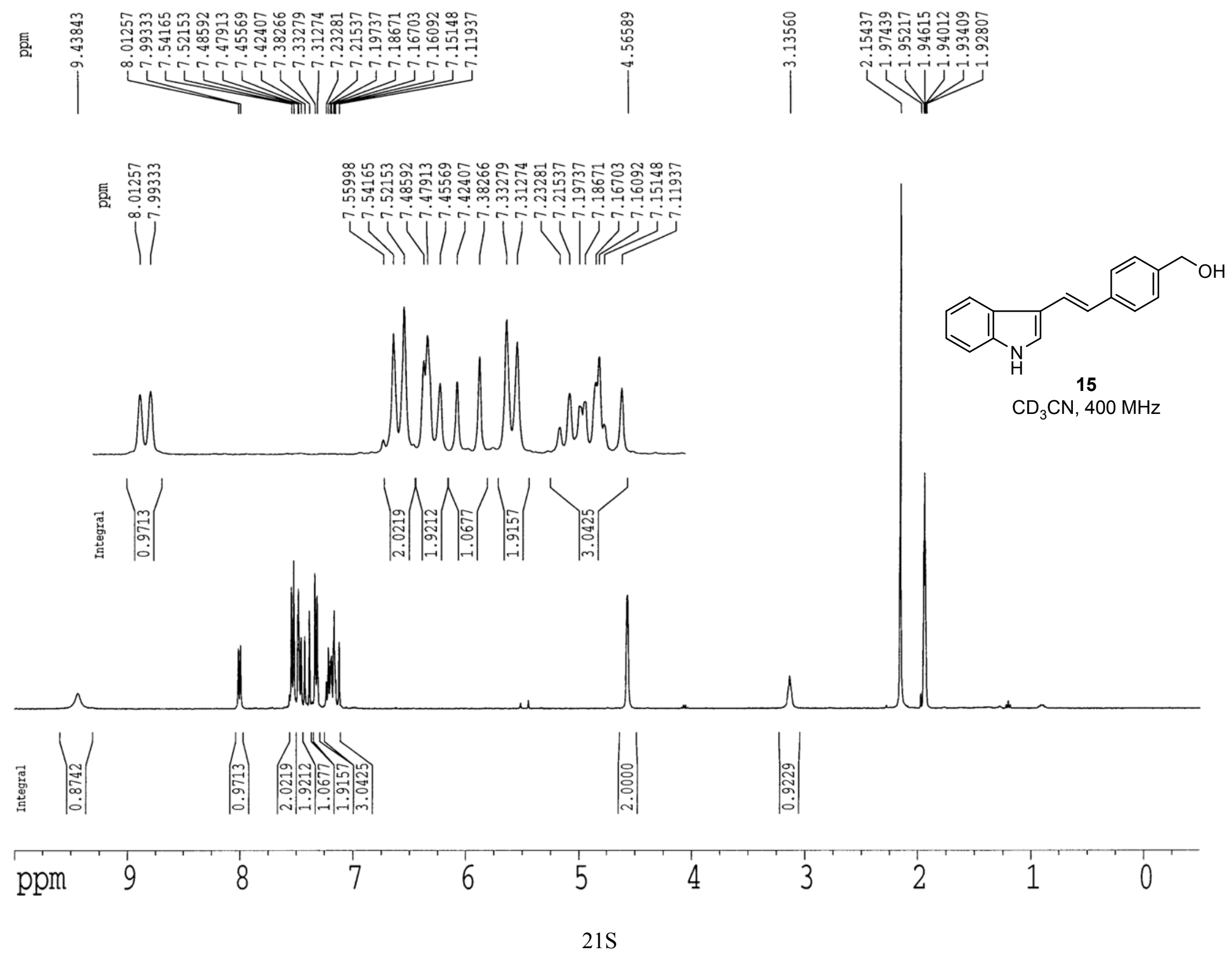


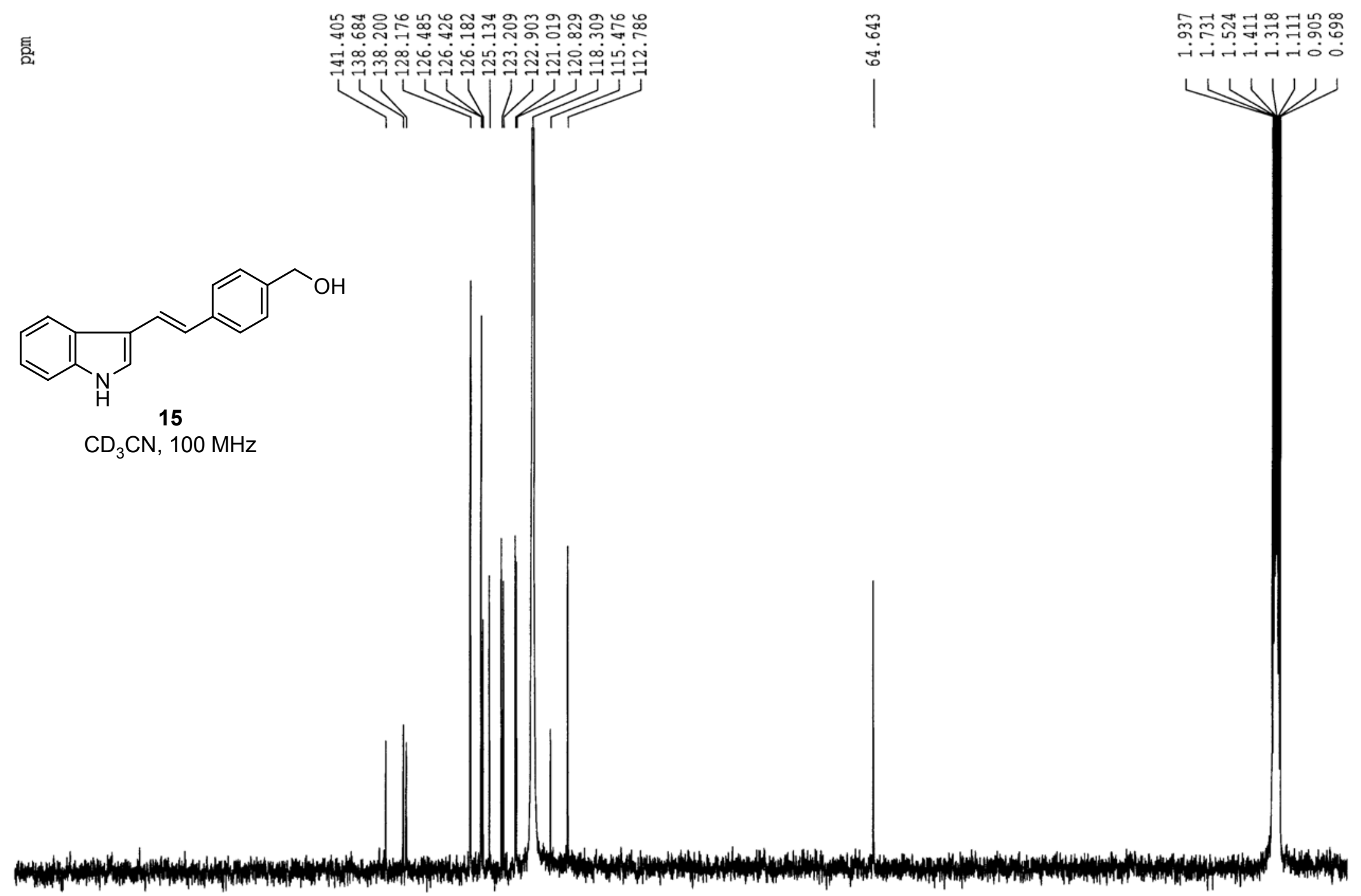

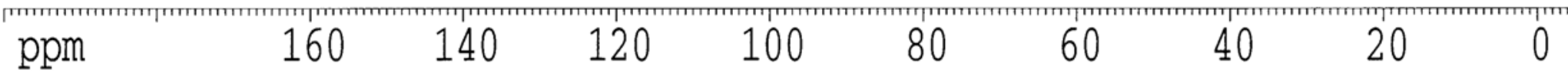




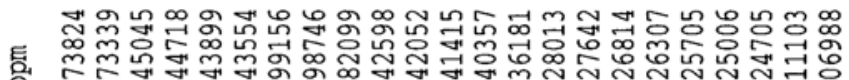

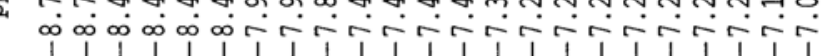

$\longrightarrow$

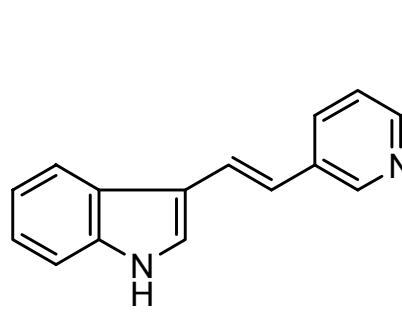

16

$\mathrm{CDCl}_{3}, 400 \mathrm{MHz}$

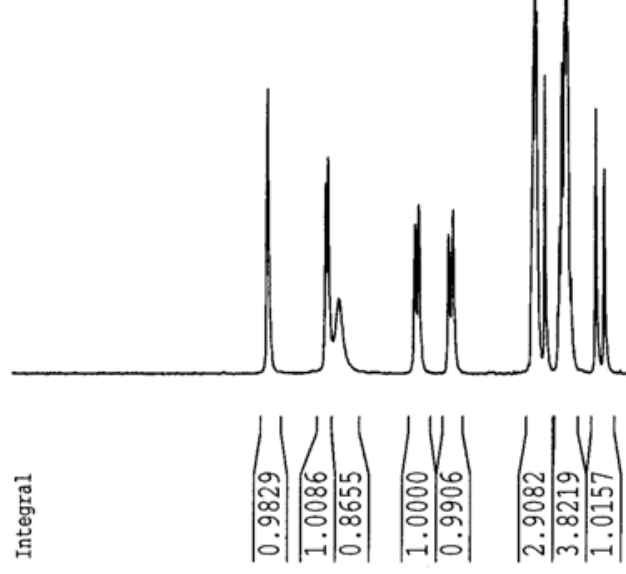

8

镸

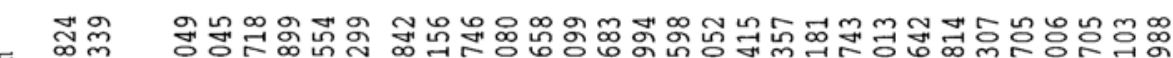

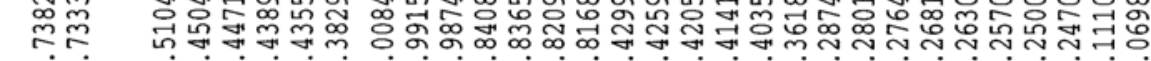

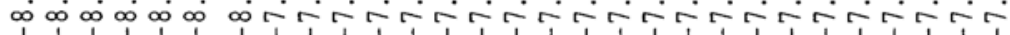
V

$1 N_{1}^{\infty}$
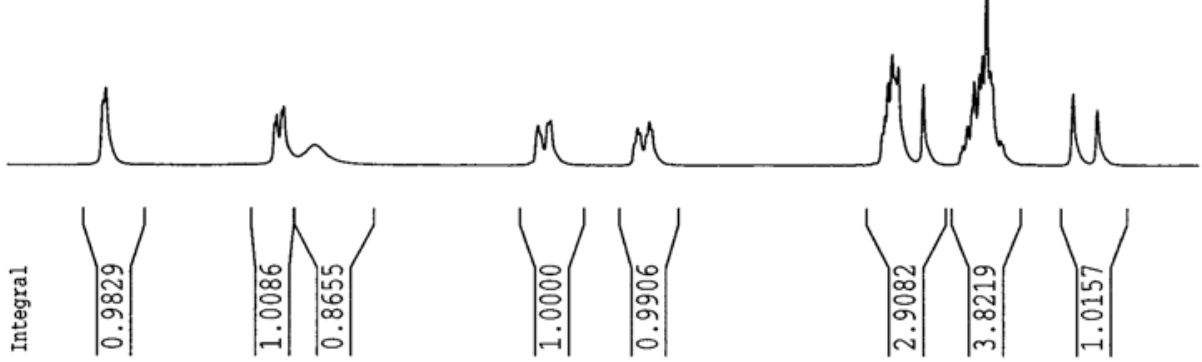

ppm 9

7

6

5

4

3

2

1 

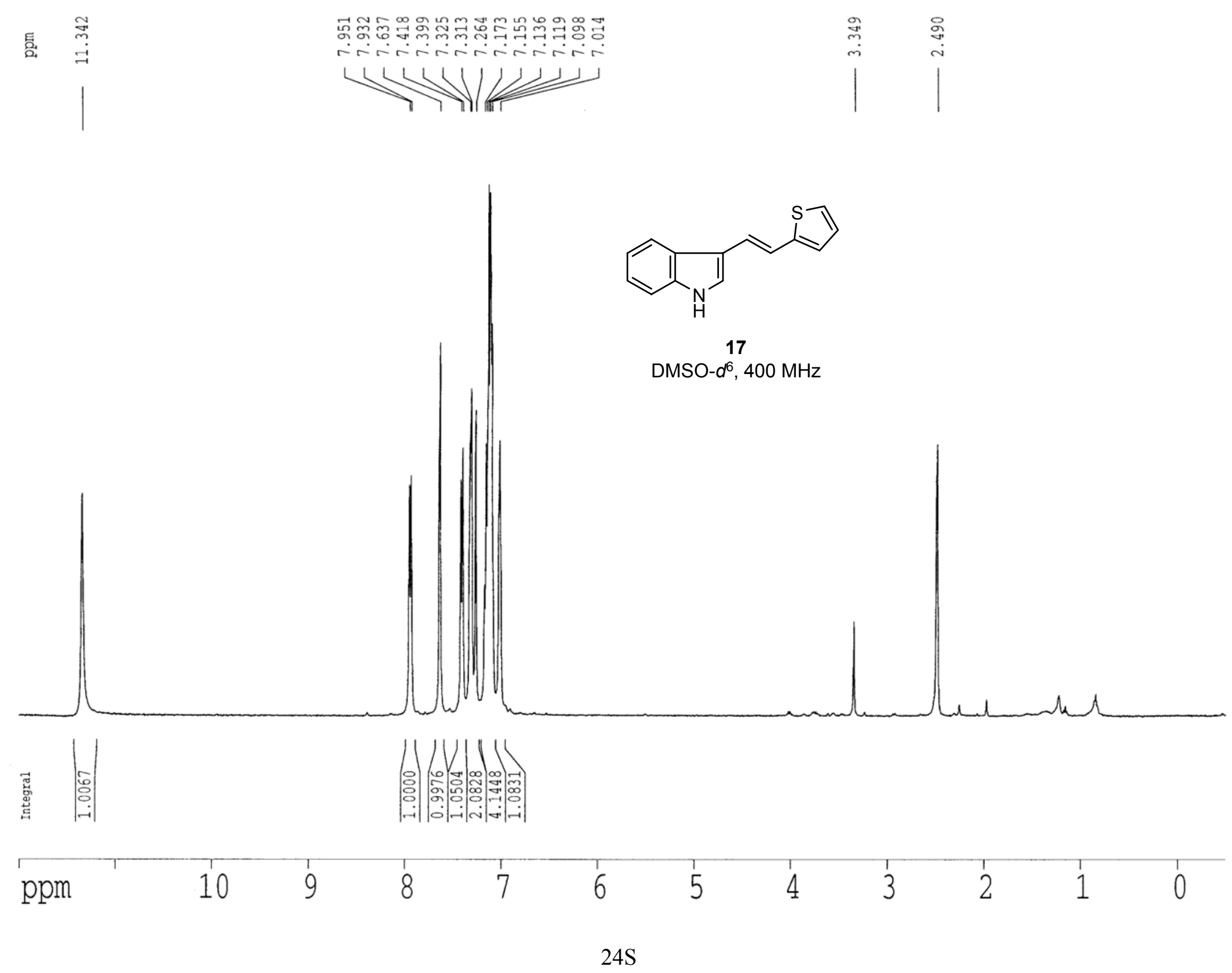
거옫ํำ웅

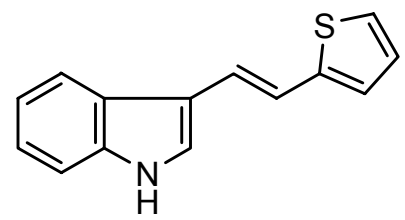

17

DMSO-d $d^{6}, 100 \mathrm{MHz}$

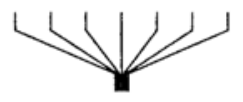

$1+\|\|\|\| \|$

\begin{tabular}{|c|c|c|c|c|c|c|c|c|c|c|}
\hline $\mathrm{ppm}$ & 180 & 160 & 140 & 120 & 100 & 80 & 60 & 40 & 20 & 0 \\
\hline
\end{tabular}



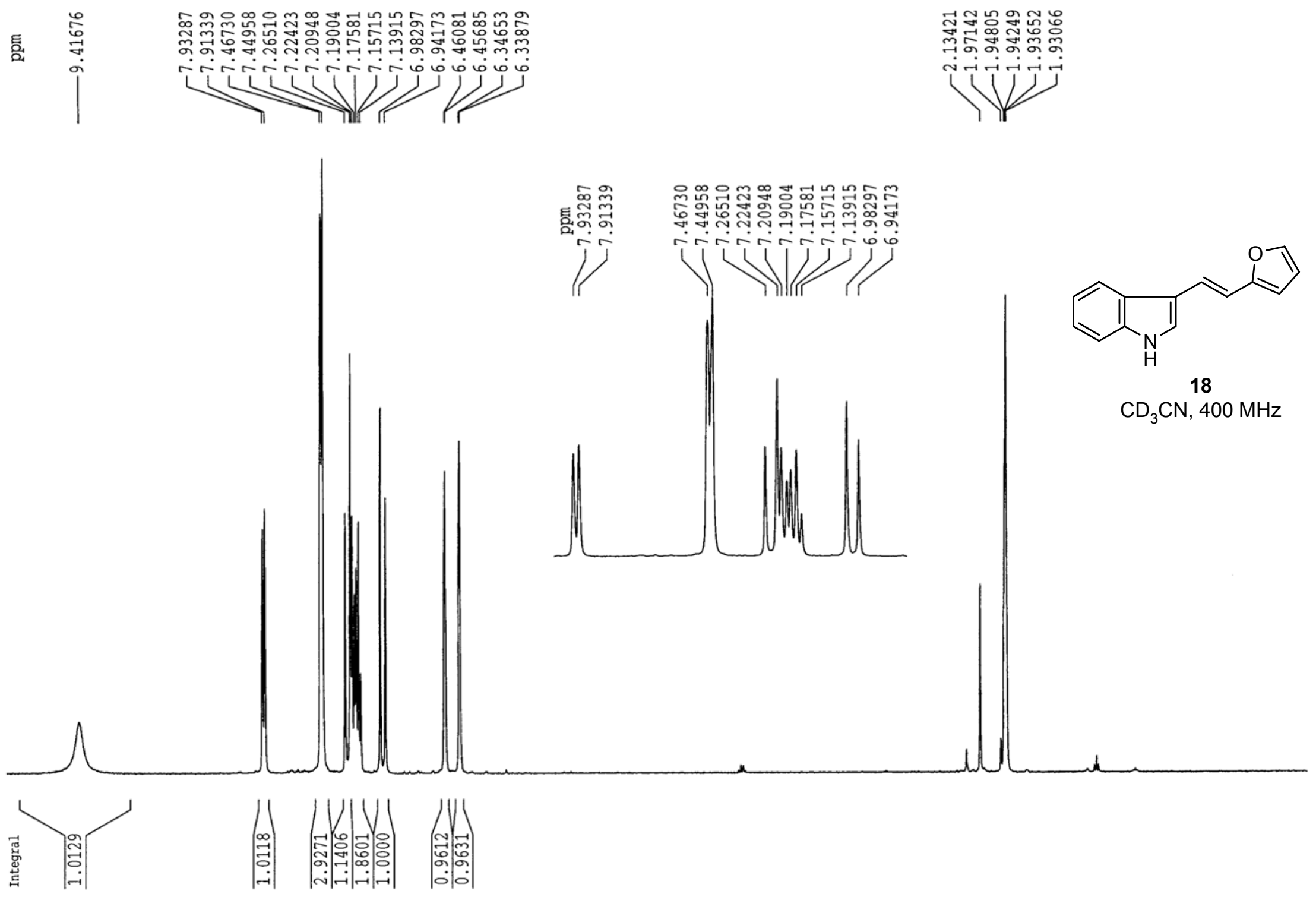

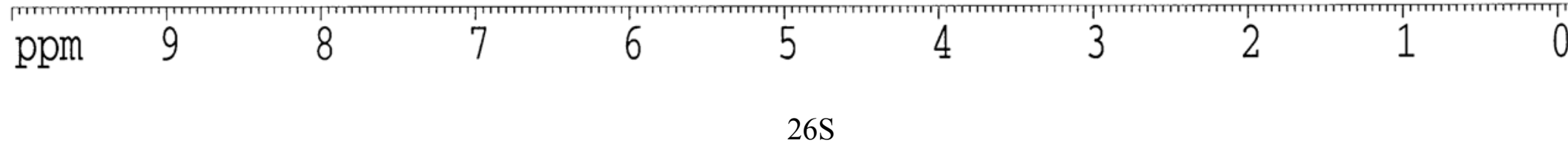



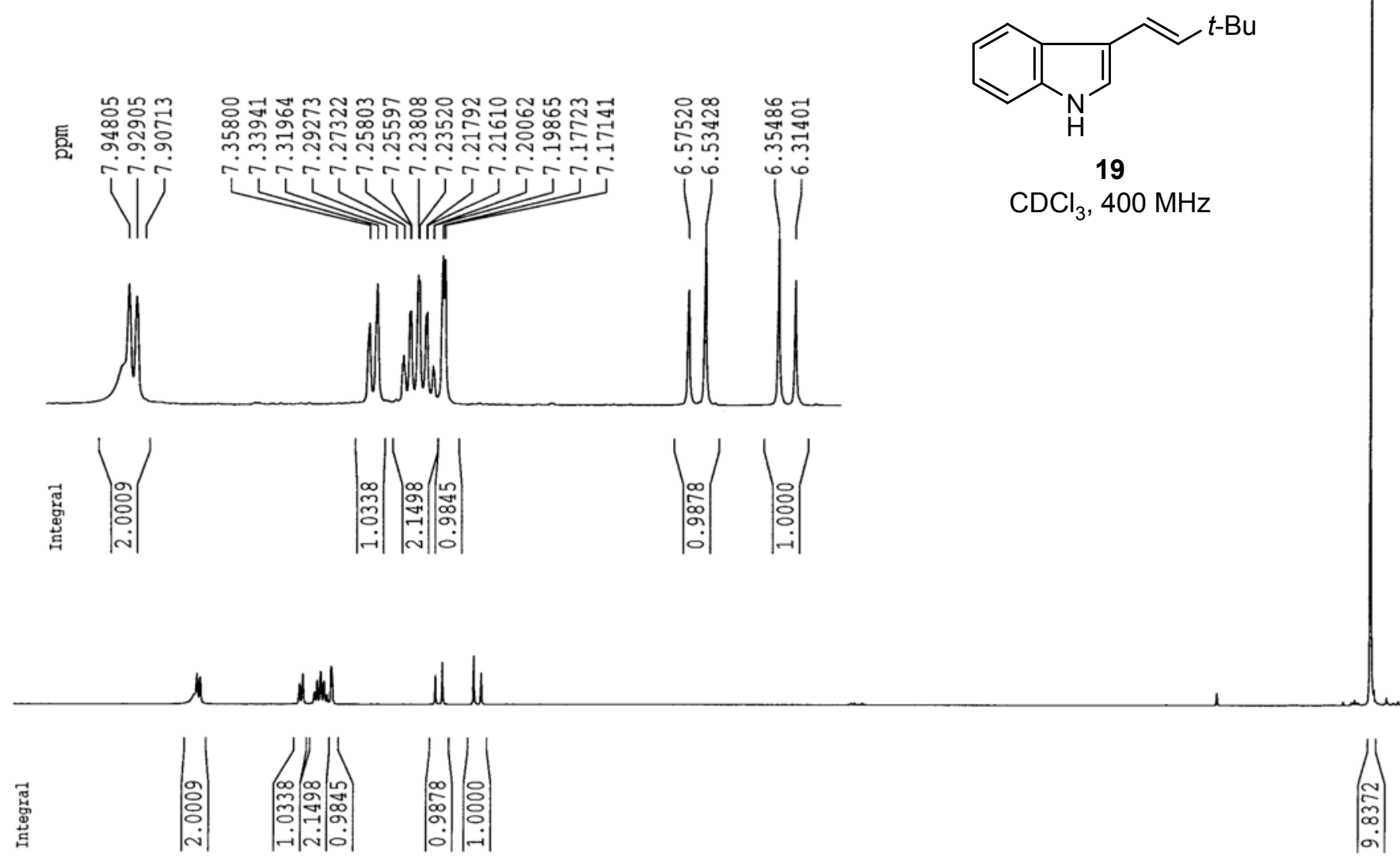

\begin{tabular}{llllllllll}
\hline $\mathrm{ppm}$ & 8 & 7 & 6 & 5 & 4 & 3 & 1 & 1 & 0 \\
& & & & & & &
\end{tabular}




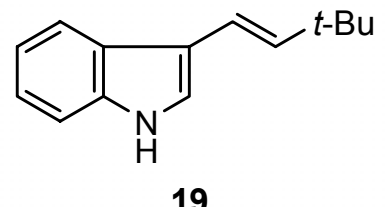

19

$\mathrm{CDCl}_{3}, 100 \mathrm{MHz}$

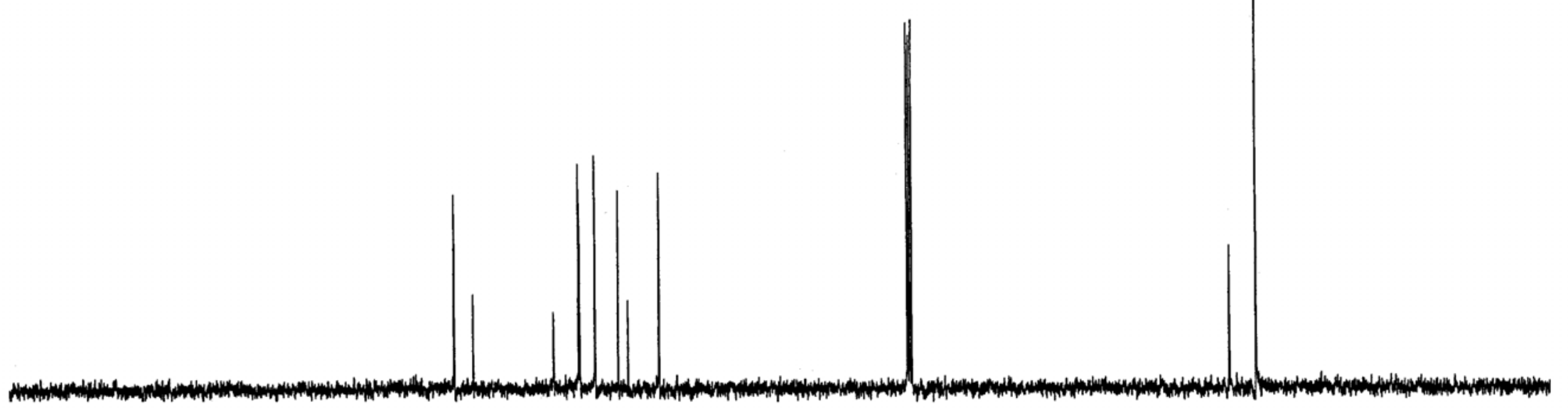

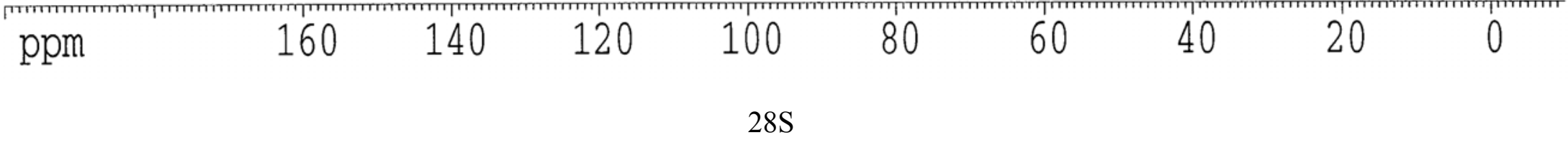




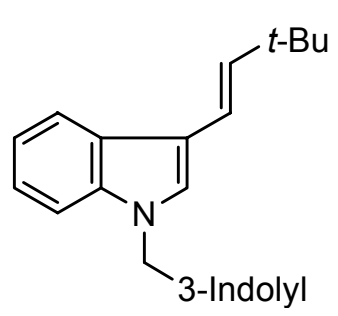

$\mathrm{CDCl}_{3}, 400 \mathrm{MHz}$
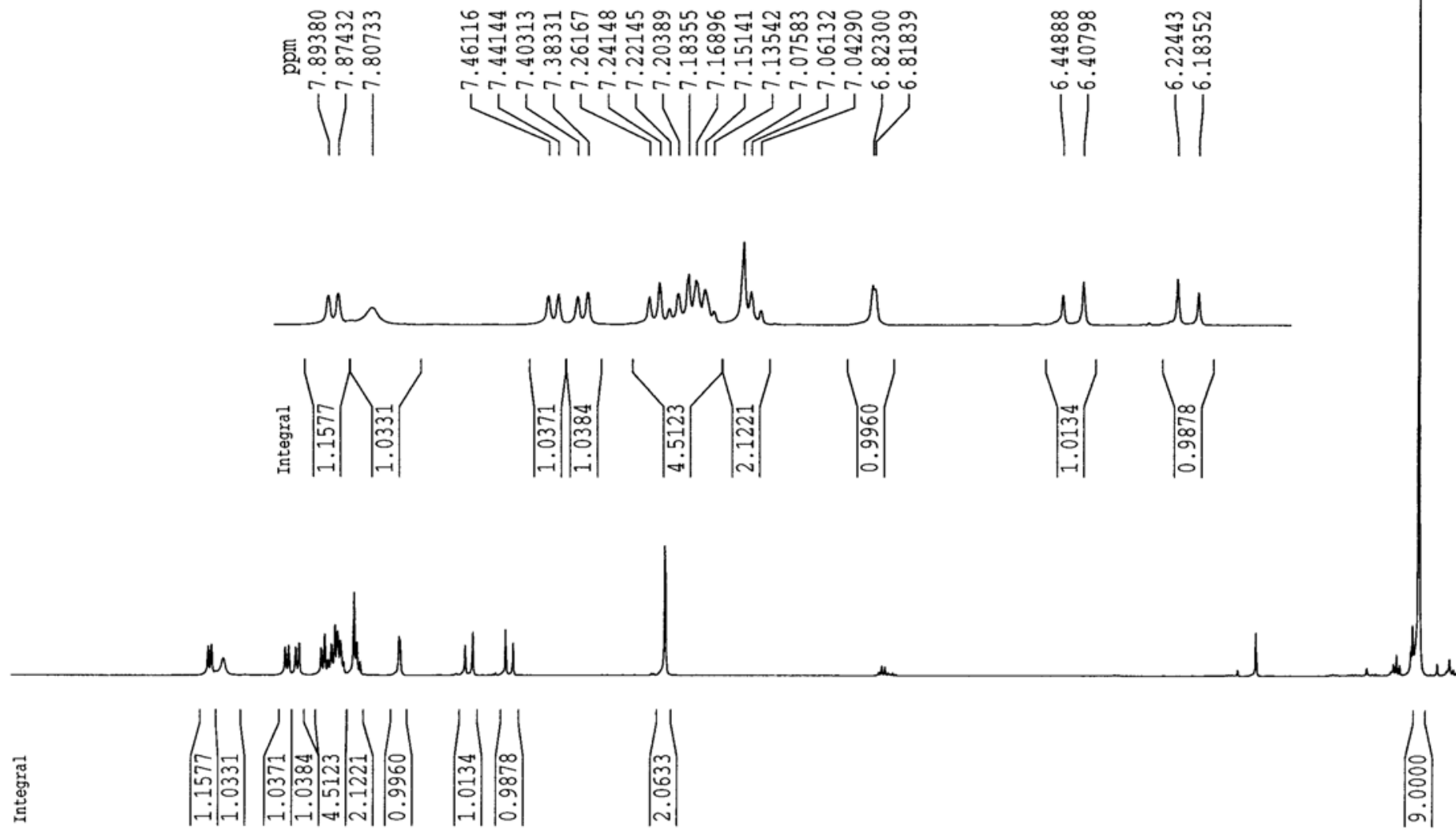

\begin{tabular}{llllllllll}
\hline $\mathrm{ppm}$ & 8 & 7 & 6 & 5 & 4 & 3 & 2 & 1 & 0 \\
& & & $29 \mathrm{~S}$ & & &
\end{tabular}




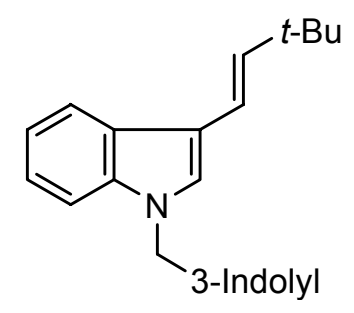

$\mathrm{CDCl}_{3}, 100 \mathrm{MHz}$

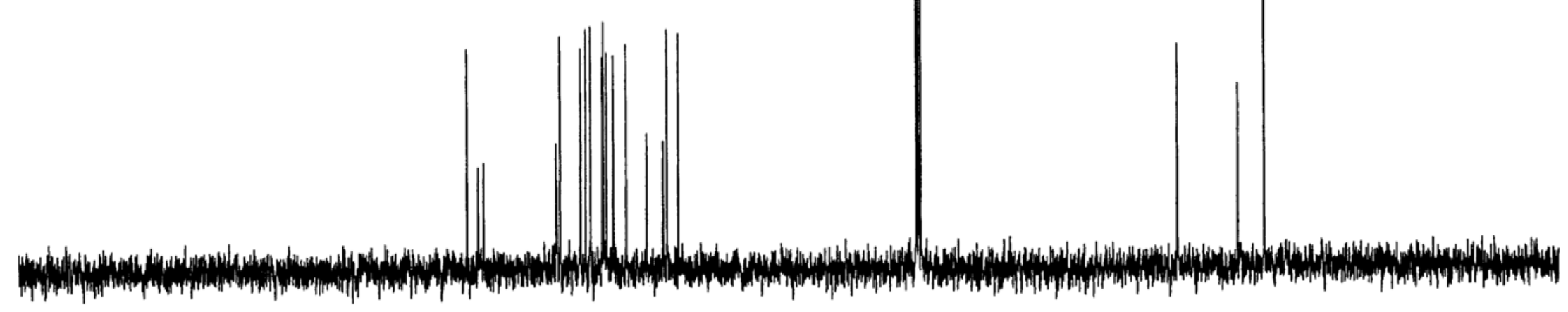

$\begin{array}{cccccccccc}\mathrm{ppm} & 160 & 140 & 120 & 100 & 80 & 60 & 40 & 20 & 0 \\ 30 \mathrm{~s} & \end{array}$




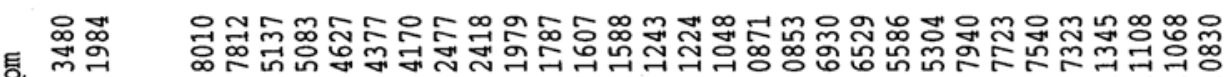

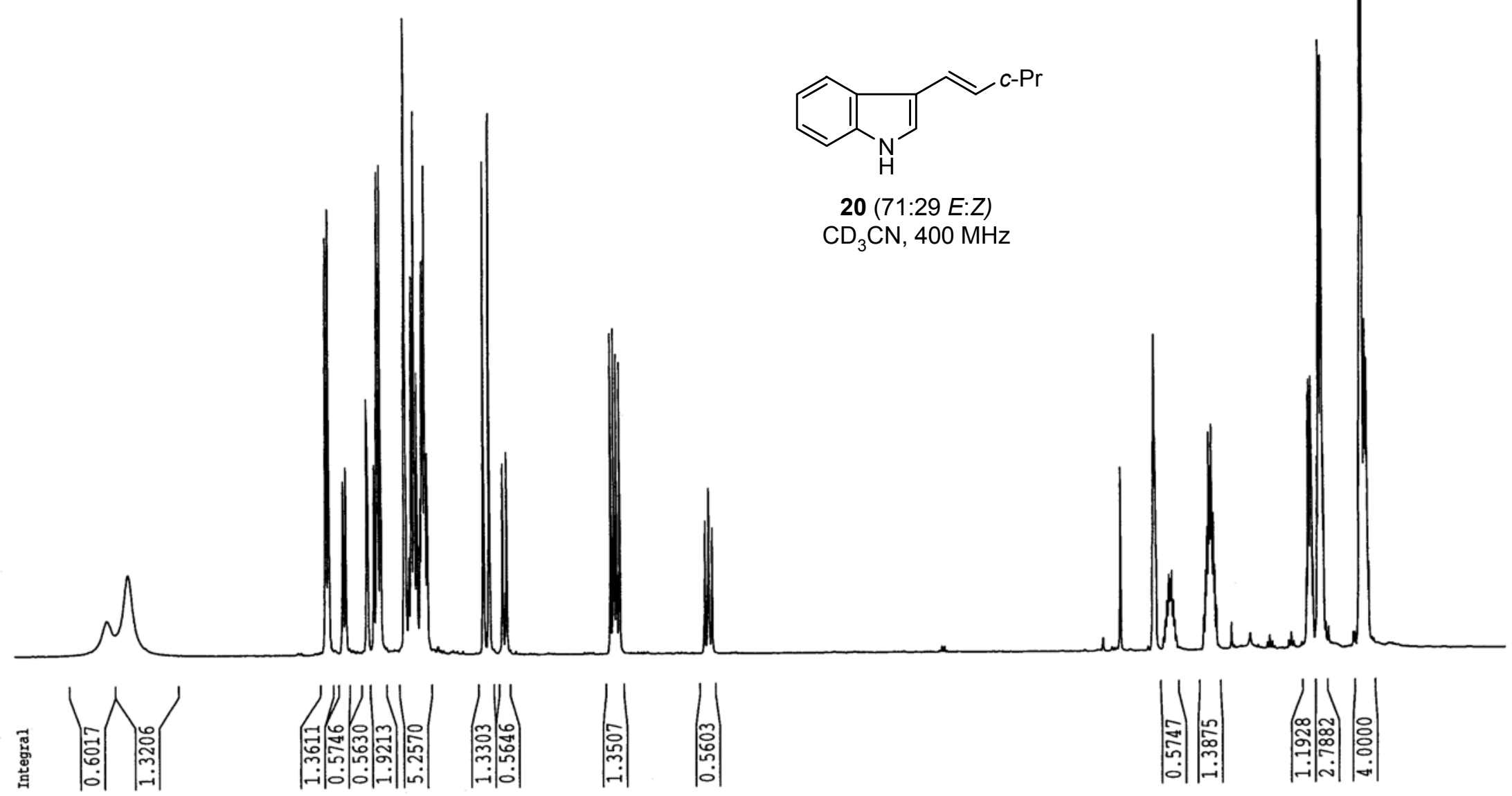

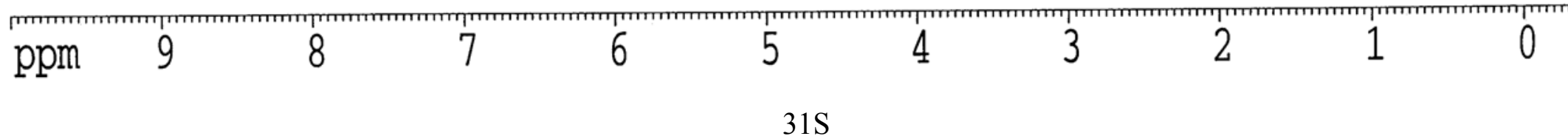



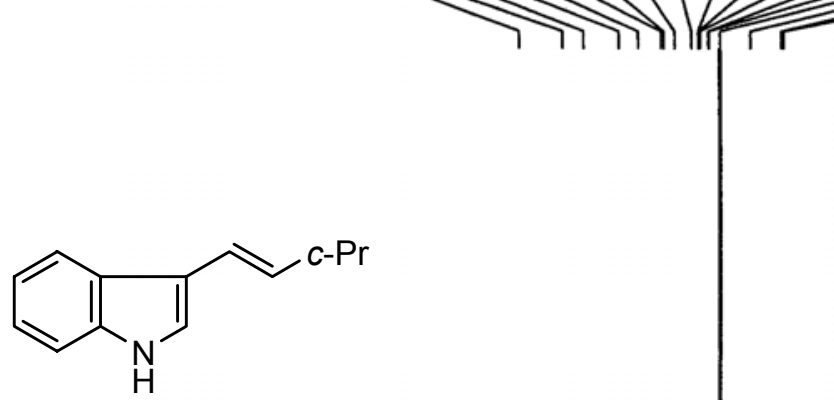

20 (71:29 E:Z)

$\mathrm{CD}_{3} \mathrm{CN}, 100 \mathrm{MHz}$
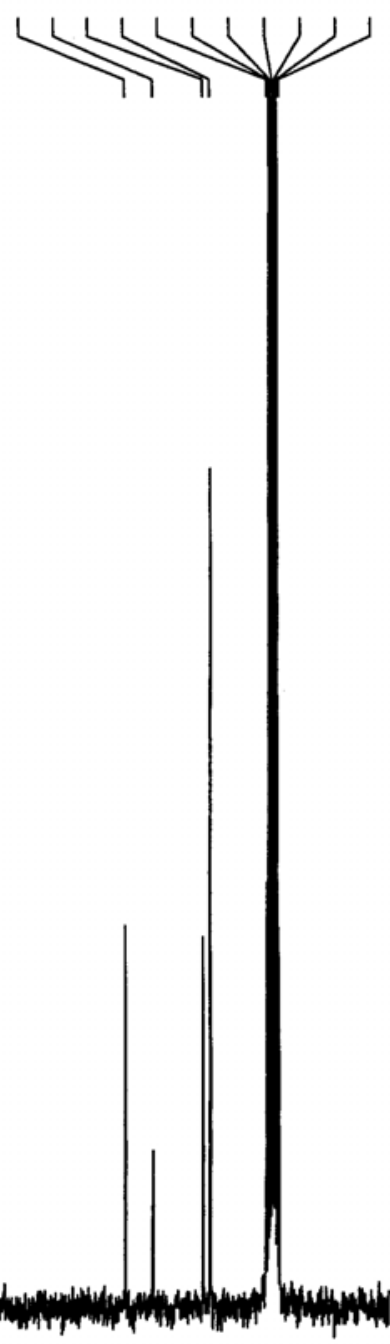

$\begin{array}{cccccccccc}\text { ppm } & 160 & 140 & 120 & 100 & 80 & 60 & 40 & 20 & 0 \\ 32 \mathrm{~S} & & & \end{array}$



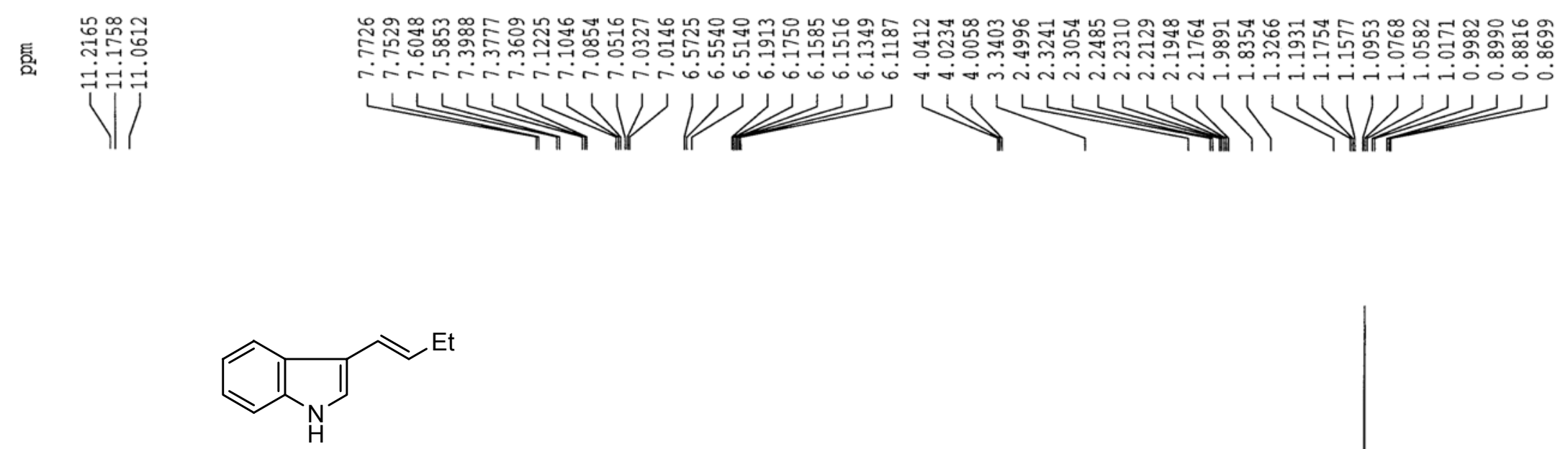

21 (86:14 E:Z)

DMSO- $d^{6}, 400 \mathrm{MHz}$

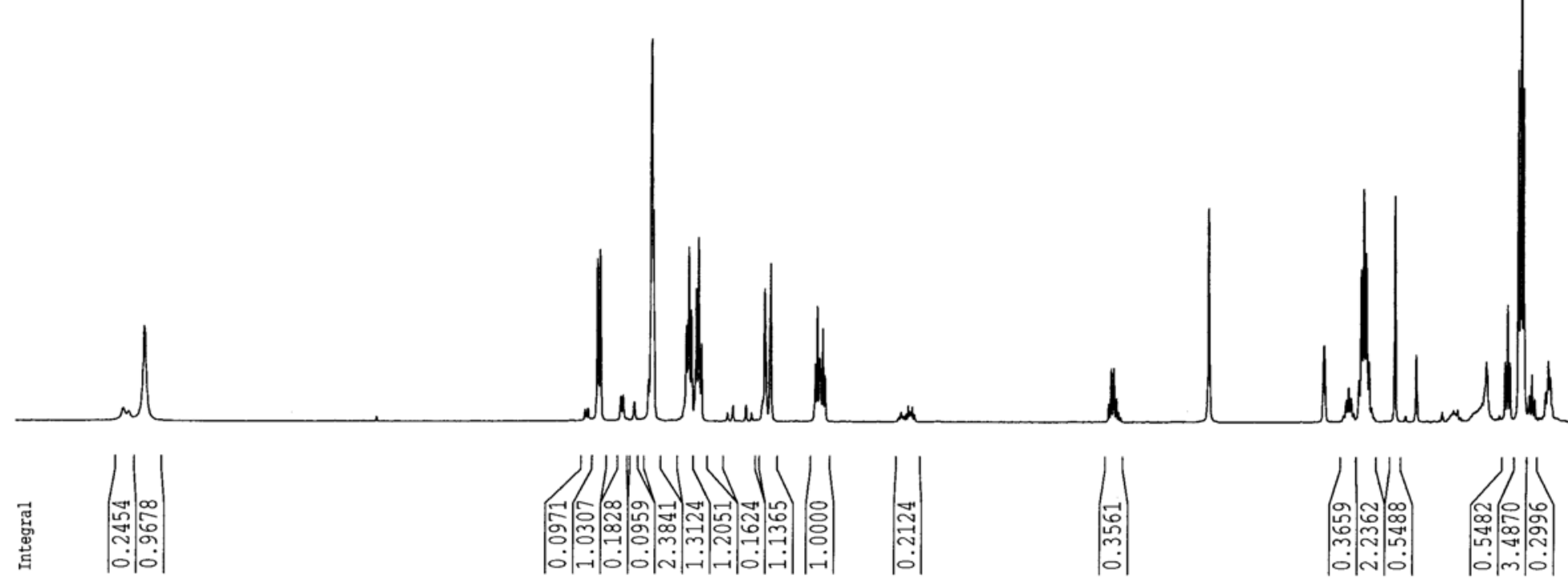

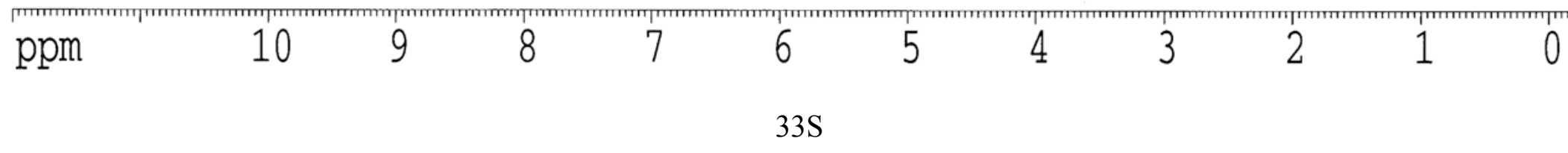




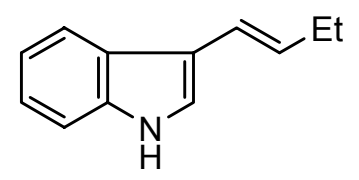

$\underset{\substack{a \\ \stackrel{-}{7}}}{\stackrel{9}{7}}$
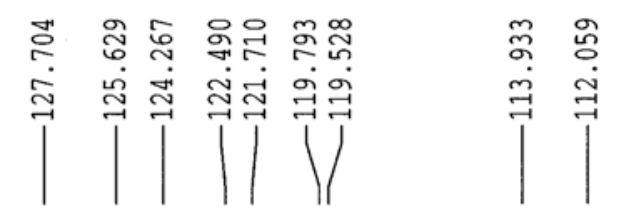

21 (86:14 E:Z)

DMSO-d, $100 \mathrm{MHz}$
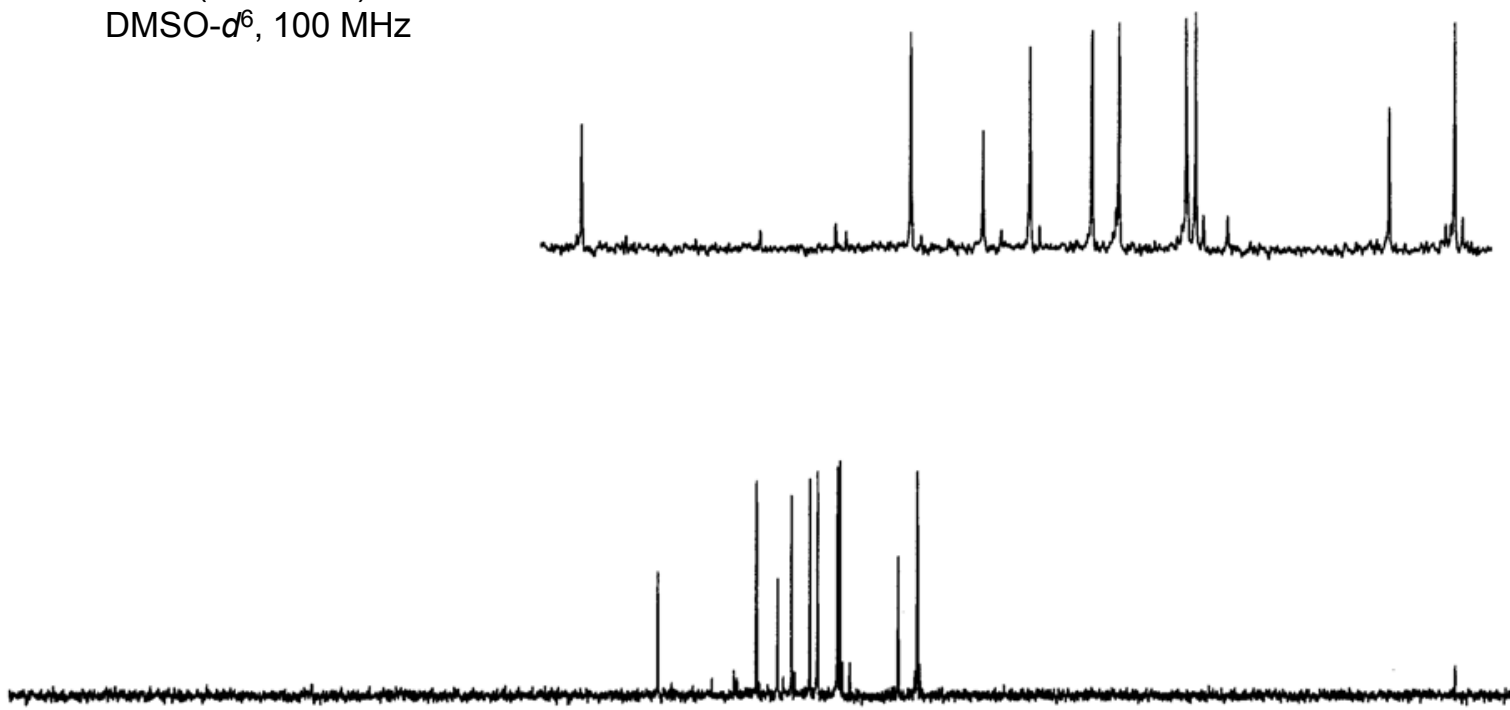

ppm 160

140

120

100

80

60

40

20

0 


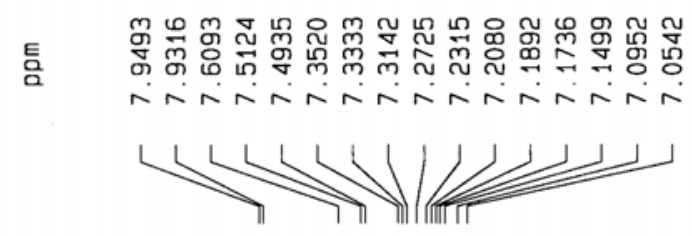

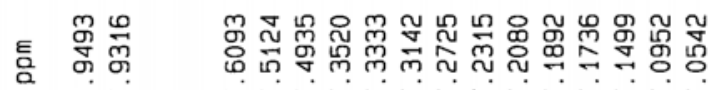
$\hat{\imath} \hat{\jmath}$

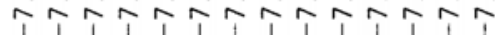
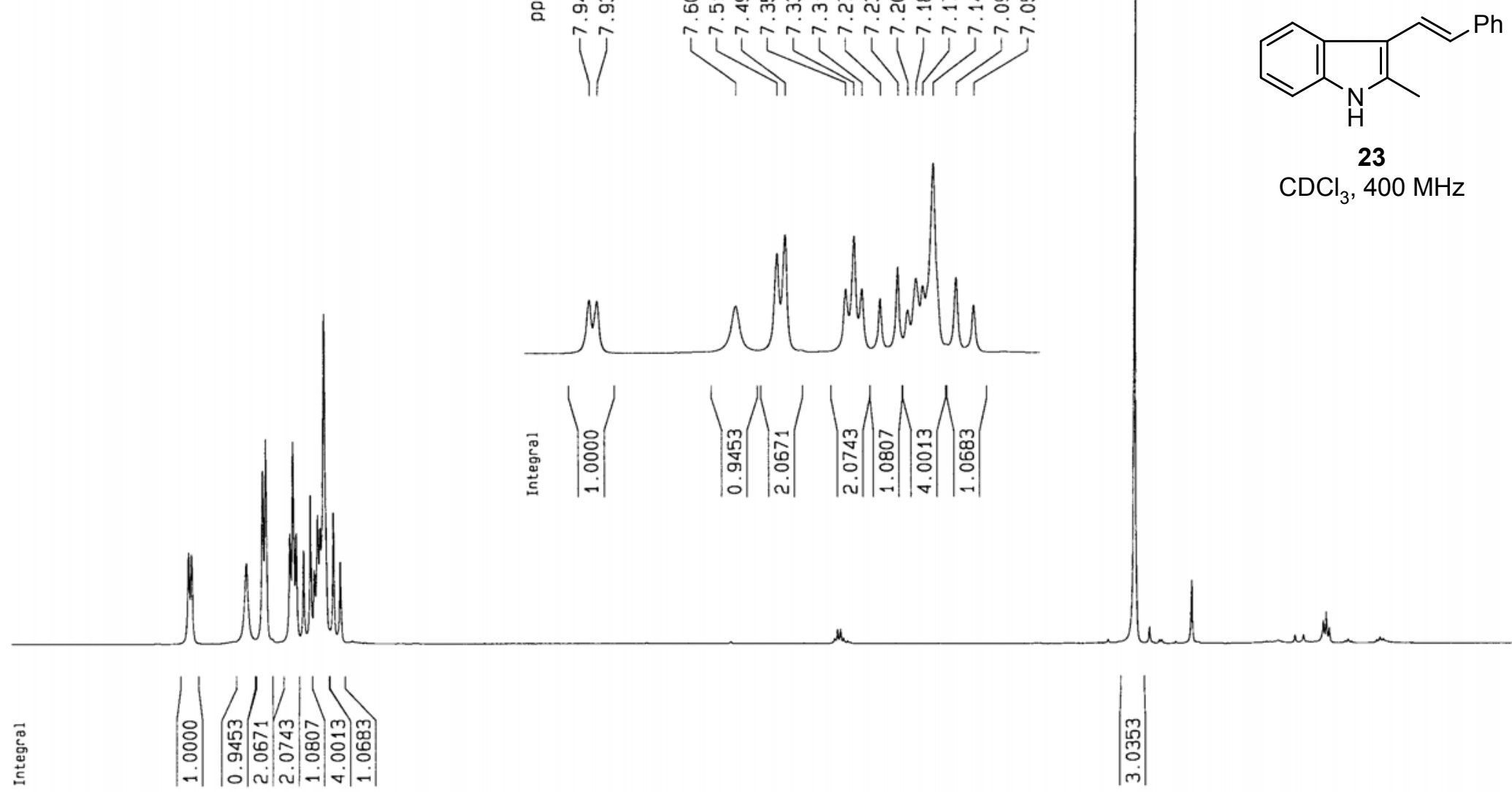
$\mathrm{CDCl}_{3}, 400 \mathrm{MHz}$

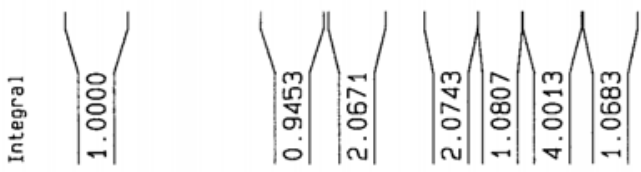

ppm 8

7

6

5

4

3

2

1 
<smiles>Cc1[nH]c2ccccc2c1/C=C/c1ccccc1</smiles>

23

$\mathrm{CDCl}_{3}, 100 \mathrm{MHz}$

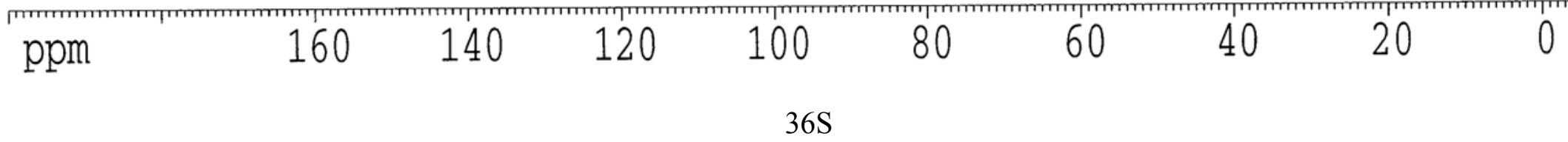

